\title{
Rate Region of the Quadratic Gaussian Two-Encoder Source-Coding Problem
}

\author{
Aaron B. Wagner*, Saurabha Tavildar ${ }^{\dagger}$ and Pramod Viswanath ${ }^{\ddagger}$
}

June 19, 2007

\begin{abstract}
We determine the rate region of the quadratic Gaussian two-encoder source-coding problem. This rate region is achieved by a simple architecture that separates the analog and digital aspects of the compression. Furthermore, this architecture requires higher rates to send a Gaussian source than it does to send any other source with the same covariance. Our techniques can also be used to determine the sum rate of some generalizations of this classical problem. Our approach involves coupling the problem to a quadratic Gaussian "CEO problem."
\end{abstract}

Keywords: multiterminal source coding, vector quantization, Gaussian sources, rate region, worst-case source, remote source, CEO problem.

\section{Introduction}

This paper addresses the quadratic Gaussian two-encoder source-coding problem, the setup for which is depicted in Fig. 1. Two encoders observe different components of a memoryless, Gaussian, vector-valued source. The encoders, without cooperating, compress their observations and send messages to a single decoder over rate-constrained, noiseless channels. The decoder attempts to reproduce both observations, subject to separate constraints on the time-averaged expected squared error of the two estimates. We seek to determine the set of rate pairs $\left(R_{1}, R_{2}\right)$ that allow us to meet a given pair of target distortions. We call this set the rate region. Of course, this problem can also be formulated for general sources and distortion measures. Our focus on the quadratic Gaussian case is motivated by its fundamental nature, its importance in applications, and its well-known extremal properties.

\footnotetext{
${ }^{*}$ School of Electrical and Computer Engineering, Cornell University. Email: wagner@ece. cornell. edu.

${ }^{\dagger}$ QUALCOMM Flarion Technologies. Email: tavildar@uiuc.edu.

¥Department of Electrical and Computer Engineering and Coordinated Science Laboratory, University of Illinois at Urbana-Champaign. Email: pramodv@uiuc.edu. Most of this work was conducted while the first two authors were with the University of Illinois.
} 
This problem is naturally viewed as a quadratic Gaussian version of Slepian and Wolf's problem [1]. Slepian and Wolf studied the problem in which the source is discrete and the decoder must reproduce it with negligible probability of error. Their celebrated result characterizes the rate region for this setup. One consequence of this characterization is that permitting the encoders to cooperate or view each other's observations does not offer any advantage, at least as far as the sum rate is concerned.

There is a natural way to harness Slepian and Wolf's result in the Gaussian setting. Each encoder first vector quantizes (VQs) its observation using a Gaussian test channel as in single-encoder rate-distortion theory. This results in two correlated digital messages, which are suitable for compression via Slepian-Wolf encoding. The decoder decodes the quantized values and estimates the source by computing a conditional expectation. This approach separates the analog and digital aspects of the compression, as shown in Fig. 2.

Our main result is an explicit characterization of the rate region for this problem. This result has three notable consequences:

(i) The architecture depicted in Fig. 2 is optimal.

(ii) This architecture requires higher rates to send a Gaussian source than it does to send any other source with the same covariance. In particular, a Gaussian source has the smallest rate region for a given covariance.

(iii) Unlike in the Slepian-Wolf problem, here decentralized encoding requires a strictly higher rate compared to centralized encoding.

The problem of determining the rate region for this setup has been open for some time [2]. Early work [2,3] used the architecture described above to prove an inner bound. Zamir and Berger [4] showed that this inner bound is asymptotically tight in the low-distortion regime, even if the source is not Gaussian. Oohama [5] determined the rate region for the problem in which only one of the two distortion constraints is present. By interpreting this problem as a relaxation of the original problem, he obtained an outer bound for the latter. He showed that this outer bound, when combined with the inner bound, determines a portion of the boundary of the rate region. As a result of his work, showing that the inner bound is tight in the sum rate suffices to complete the characterization of the rate region. This is shown in the present paper.

Our approach is to lower bound the sum rate of a given code in two different ways. The first way amounts to considering the rate required by a hypothetical centralized encoder that achieves the same error covariance matrix as the code. The second way is to establish a connection between this problem and the quadratic Gaussian "CEO problem," for which the rate region is already known. For some codes, the cooperative bound may be tighter. For others, the CEO bound may be tighter. Taking the maximum of the two lower bounds yields a composite lower bound that is sufficiently strong to prove the desired result.

The next section contains a precise formulation of the problem and a statement of our main result, Theorem 1. In Section 3, we describe the separationbased compression architecture. There we also discuss the worst-case property 
of the Gaussian distribution. We provide the necessary background on the CEO problem and some other preliminaries to the converse proof in Section 4. The converse proof itself is contained in Section 5. In Section 6, we show how the converse proof technique can be used to determine the rate region for a more general version of the problem in which the decoder aims to reproduce certain linear combinations of the source components. In Section 7, we show how the proof technique can be extended to handle the case of more than two sources, if a certain symmetry condition holds. Section 8 contains some concluding remarks.

We use the following notation. Boldface, lower case letters $(\boldsymbol{\mu})$ denote vectors, while boldface, upper case letters $(\boldsymbol{D})$ denote matrices. Lightface letters $(\rho, R)$ denote scalars. Whether a variable is deterministic or random should be clear from the context.

\section{Problem Formulation and Main Result}

Let $\left\{\left(y_{1}^{n}(i), y_{2}^{n}(i)\right)\right\}_{i=1}^{n}$ be a sequence of independent and identically distributed (i.i.d.) Gaussian zero-mean random vectors. Let

$$
\boldsymbol{K}_{y}=\left[\begin{array}{ll}
1 & \rho \\
\rho & 1
\end{array}\right]
$$

denote the covariance matrix of $\left(y_{1}^{n}(1), y_{2}^{n}(1)\right)$. We use $y_{1}^{n}$ to denote

$$
\left\{y_{1}^{n}(i)\right\}_{i=1}^{n} \text {, }
$$

$y_{1}^{n}(j: k)$ to denote

$$
\left\{y_{1}^{n}(i)\right\}_{i=j}^{k},
$$

$\mathbf{y}^{n}(i)$ to denote $\left(y_{1}^{n}(i), y_{2}^{n}(i)\right), \mathbf{y}^{n}$ to denote $\left\{\left(y_{1}^{n}(i), y_{2}^{n}(i)\right)\right\}_{i=1}^{n}$, etc. Analogous notation will be used for other vectors that appear later.

The first encoder observes $y_{1}^{n}$, then sends a message to the decoder using a mapping

$$
f_{1}^{(n)}: \mathbb{R}^{n} \mapsto\left\{1, \ldots, M_{1}^{(n)}\right\}
$$

The second encoder operates analogously. The decoder uses the received messages to estimate both $y_{1}^{n}$ and $y_{2}^{n}$ using mappings

$$
\varphi_{j}^{(n)}:\left\{1, \ldots, M_{1}^{(n)}\right\} \times\left\{1, \ldots, M_{2}^{(n)}\right\} \mapsto \mathbb{R}^{n} \quad j=1,2 .
$$

Definition 1 (Quadratic Gaussian Two-Encoder Source Coding Problem). A rate-distortion vector $\left(R_{1}, R_{2}, d_{1}, d_{2}\right)$ is strict-sense achievable if there exists a block length $n$, encoders $f_{1}^{(n)}$ and $f_{2}^{(n)}$, and a decoder $\left(\varphi_{1}^{(n)}, \varphi_{2}^{(n)}\right)$ such that ${ }^{1}$

$$
\begin{aligned}
R_{j} & \geq \frac{1}{n} \log M_{j}^{(n)} \text { for all } j \text { in }\{1,2\}, \text { and } \\
d_{j} & \geq \frac{1}{n} \sum_{i=1}^{n} E\left[\left(y_{j}^{n}(i)-\hat{y}_{j}^{n}(i)\right)^{2}\right] \text { for all } j \text { in }\{1,2\},
\end{aligned}
$$

\footnotetext{
${ }^{1}$ All logarithms in this paper are base two.
} 
where

$$
\hat{y}_{j}^{n}=\varphi_{j}^{(n)}\left(f_{1}^{(n)}\left(y_{1}^{n}\right), f_{2}^{(n)}\left(y_{2}^{n}\right)\right) \quad j \in\{1,2\} .
$$

Let $\mathcal{R D}^{\star}$ denote the set of strict-sense achievable rate-distortion vectors. We define the set of achievable rate-distortion vectors to be the closure of $\mathcal{R D}^{\star}$,

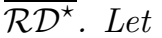

$$
\mathcal{R}^{\star}\left(d_{1}, d_{2}\right)=\left\{\left(R_{1}, R_{2}\right):\left(R_{1}, R_{2}, d_{1}, d_{2}\right) \in \overline{\mathcal{R} \mathcal{D}^{\star}}\right\} .
$$

We call $\mathcal{R}^{\star}(\cdot, \cdot)$ the rate region for the problem. The (minimum) sum rate for a given distortion pair $\left(d_{1}, d_{2}\right)$ is defined to be

$$
\inf \left\{R_{1}+R_{2}:\left(R_{1}, R_{2}\right) \in \mathcal{R}^{\star}\left(d_{1}, d_{2}\right)\right\} .
$$

We note that there is no loss of generality in assuming that $E\left[y_{1}^{2}\right]=E\left[y_{2}^{2}\right]=$ 1 , since the observations and the estimates can be scaled to reduce the general case to this one. By similar reasoning, we may assume that $\rho \geq 0$, i.e., that the observations of the two encoders are nonnegatively correlated. Since the two extreme cases $\rho=0$ and $\rho=1$ can be handled using existing techniques, we will assume throughout the remainder of the paper that $0<\rho<1$.

We now define three sets that will be used to describe the rate region. Let

$$
\mathcal{R}_{1}^{\star}\left(d_{1}\right)=\left\{\left(R_{1}, R_{2}\right): R_{1} \geq \frac{1}{2} \log ^{+}\left[\frac{1}{d_{1}}\left(1-\rho^{2}+\rho^{2} 2^{-2 R_{2}}\right)\right]\right\}
$$

where $\log ^{+} x=\max (\log x, 0)$. Likewise, let

$$
\mathcal{R}_{2}^{\star}\left(d_{2}\right)=\left\{\left(R_{1}, R_{2}\right): R_{2} \geq \frac{1}{2} \log ^{+}\left[\frac{1}{d_{2}}\left(1-\rho^{2}+\rho^{2} 2^{-2 R_{1}}\right)\right]\right\} .
$$

Finally, let

$$
\mathcal{R}_{\text {sum }}^{\star}\left(d_{1}, d_{2}\right)=\left\{\left(R_{1}, R_{2}\right): R_{1}+R_{2} \geq \frac{1}{2} \log ^{+}\left[\frac{\left(1-\rho^{2}\right) \beta\left(d_{1}, d_{2}\right)}{2 d_{1} d_{2}}\right]\right\},
$$

where

$$
\beta\left(d_{1}, d_{2}\right)=1+\sqrt{1+\frac{4 \rho^{2} d_{1} d_{2}}{\left(1-\rho^{2}\right)^{2}}} .
$$

Later we will see that we can often interpret the the logarithm in the definition of $\mathcal{R}_{\text {sum }}^{\star}(\cdot, \cdot)$ as a mutual information

$$
\frac{1}{2} \log \frac{\left|\boldsymbol{K}_{y}\right|}{\left|\boldsymbol{D}^{*}\right|}
$$

where $\boldsymbol{D}^{*}$ is the covariance matrix of the errors $\left(y_{1}-\hat{y}_{1}, y_{2}-\hat{y}_{2}\right)$ in a sum-rate optimal code. Throughout the paper we assume that all distortion constraints $\left(d_{1}\right.$ and $d_{2}$ in this case) are positive.

Theorem 1. For the Gaussian two-encoder source-coding problem,

$$
\mathcal{R}^{\star}\left(d_{1}, d_{2}\right)=\mathcal{R}_{1}^{\star}\left(d_{1}\right) \cap \mathcal{R}_{2}^{\star}\left(d_{2}\right) \cap \mathcal{R}_{\text {sum }}^{\star}\left(d_{1}, d_{2}\right) .
$$


An example of the rate region is shown in Fig. 3. The direct part of this result was previously known and is discussed in the next section. It was also previously known that the rate region was contained in the set $\mathcal{R}_{1}^{\star}\left(d_{1}\right) \cap \mathcal{R}_{2}^{\star}\left(d_{2}\right)$. Our contribution is a proof that the rate region in contained in $\mathcal{R}_{\text {sum }}^{\star}\left(d_{1}, d_{2}\right)$. This is provided in Sections 4 and 5. Sections 6 and 7 present some extensions of this result to problems with more general distortion constraints and more than two sources, respectively.

\section{Direct Part and Worst-Case Property}

Translating the architecture in Fig. 2 into an inner bound on the rate region is a straightforward exercise in network information theory. Since proofs of similar bounds are available $[2,6,7,8,9]$, we provide only a high-level view of the proof here.

Let $\mathcal{U}\left(d_{1}, d_{2}\right)$ denote the set of real-valued random variables $u_{1}$ and $u_{2}$ such that

(i) $u_{1} \leftrightarrow y_{1} \leftrightarrow y_{2} \leftrightarrow u_{2}$, meaning that $u_{1}, y_{1}, y_{2}$, and $u_{2}$ form a Markov chain in this order ${ }^{2}$, and

(ii) $E\left[\left(y_{j}-E\left[y_{j} \mid \mathbf{u}\right]\right)^{2}\right] \leq d_{j}$ for $j \in\{1,2\}$.

Then fix $\mathbf{u}$ in $\mathcal{U}\left(d_{1}, d_{2}\right)$ and a large integer $n$. By the proof of the point-to-point rate-distortion theorem, the first vector quantizer can send $I\left(y_{1} ; u_{1}\right)$ bits per sample to the first Slepian-Wolf encoder that convey a string $u_{1}^{n}$ that is jointly typical with $y_{1}^{n}$ with high probability. Likewise, the second vector quantizer can use $I\left(y_{2} ; u_{2}\right)$ bits per sample to send its Slepian-Wolf encoder a string $u_{2}^{n}$ that is jointly typical with $y_{2}^{n}$ with high probability.

The Slepian-Wolf encoders could view the quantized strings $u_{1}^{n}$ and $u_{2}^{n}$ as individual symbols from a digital source to be compressed [9]. They would then accumulate many such symbols to compress. Alternatively, one can apply the arguments behind the Slepian and Wolf theorem directly to $u_{1}^{n}$ and $u_{2}^{n}[2,6,7,8]$. Either way, the decoder can recover $u_{1}^{n}$ and $u_{2}^{n}$ so long as

$$
\begin{aligned}
R_{1} & \geq I\left(y_{1} ; u_{1} \mid u_{2}\right) \\
R_{2} & \geq I\left(y_{2} ; u_{2} \mid u_{1}\right) \\
R_{1}+R_{2} & \geq I(\mathbf{y} ; \mathbf{u}) .
\end{aligned}
$$

The decoder can then in principle compute the minimum mean-squared error (MMSE) estimate of $\mathbf{y}^{n}$ given $\mathbf{u}^{n}$, and (ii) above guarantees that this estimate will comply with the distortion constraints. By a time-sharing argument, one can show that the rate region is convex. This outlines the proof of the following inner bound.

\footnotetext{
${ }^{2}$ This condition is sometimes called the "long Markov chain" [4].
} 
Proposition 1 (Berger-Tung Inner Bound [2, 3]). The separation-based architecture achieves the rates

$$
\begin{gathered}
\mathcal{R}^{i}\left(d_{1}, d_{2}\right)=\left\{\left(R_{1}, R_{2}\right): \text { there exists } \mathbf{u} \in \mathcal{U}\left(d_{1}, d_{2}\right)\right. \text { such that } \\
R_{1} \geq I\left(y_{1} ; u_{1} \mid u_{2}\right) \\
R_{2} \geq I\left(y_{2} ; u_{2} \mid u_{1}\right) \\
\left.R_{1}+R_{2} \geq I(\mathbf{y} ; \mathbf{u})\right\} .
\end{gathered}
$$

In particular, the rate region contains the convex hull of this set.

It is unclear a priori how to compute this inner bound. A natural approach is to place additional constraints on $\mathbf{u}$ to create a potentially smaller inner bound that is amenable to explicit calculation. Let $\mathcal{U}_{G}\left(d_{1}, d_{2}\right)$ denote the set of $\mathbf{u}$ in $\mathcal{U}\left(d_{1}, d_{2}\right)$ such that $u_{j}$ has zero mean and unit variance for each $j$, and there exists a random vector $\mathbf{z}$ such that

(i) For some constants $c_{1}$ and $c_{2}$ in $[0,1)$,

$$
\begin{aligned}
& u_{1}=c_{1} y_{1}+z_{1} \\
& u_{2}=c_{2} y_{2}+z_{2},
\end{aligned}
$$

(ii) $\mathbf{z}$ is Gaussian and its components are independent,

(iii) $\mathbf{z}$ is independent of $\mathbf{y}$,

(v) $E\left[\left(y_{j}-E\left[y_{j} \mid \mathbf{u}\right]\right)^{2}\right] \leq d_{j}$ for all $j$ in $\{1,2\}$.

We will refer to a random vector $\mathbf{u}$ satisfying conditions (i)-(iii) as a distributed Gaussian test channel or, when there is no ambiguity, as simply a test channel. Note that the set of distributed Gaussian test channels is parametrized by $c_{1}$ and $c_{2}$.

Let

$$
\begin{gathered}
\mathcal{R}_{G}^{i}\left(d_{1}, d_{2}\right)=\left\{\left(R_{1}, R_{2}\right): \text { there exists } \mathbf{u} \in \mathcal{U}_{G}\left(d_{1}, d_{2}\right)\right. \text { such that } \\
R_{1} \geq I\left(y_{1} ; u_{1} \mid u_{2}\right) \\
R_{2} \geq I\left(y_{2} ; u_{2} \mid u_{1}\right) \\
\left.R_{1}+R_{2} \geq I(\mathbf{y} ; \mathbf{u})\right\} .
\end{gathered}
$$

Lemma 1. The separation-based architecture achieves $\mathcal{R}_{G}^{i}\left(d_{1}, d_{2}\right)$, which satisfies

$$
\mathcal{R}_{G}^{i}\left(d_{1}, d_{2}\right)=\mathcal{R}_{1}^{\star}\left(d_{1}\right) \cap \mathcal{R}_{2}^{\star}\left(d_{2}\right) \cap \mathcal{R}_{\text {sum }}^{\star}\left(d_{1}, d_{2}\right) .
$$

This lemma is an immediate consequence of Proposition 1, except for the equality in (4). Later, we state and prove a more general version of this equality (Lemma 7 in Section 6). Since one can verify directly that $\mathcal{R}_{1}^{\star}\left(d_{1}\right), \mathcal{R}_{2}^{\star}\left(d_{2}\right)$, and $\mathcal{R}_{\text {sum }}^{\star}\left(d_{1}, d_{2}\right)$ are convex, it follows that $\mathcal{R}_{G}^{i}\left(d_{1}, d_{2}\right)$ is also convex. Thus, time sharing will not enlarge this region. In the remainder of the paper, whenever we 
consider the separation-based architecture, we will assume that $\mathbf{u}$ is a distributed Gaussian test channel.

Theorem 1 and Lemma 1 together show that $\mathcal{R}_{G}^{i}\left(d_{1}, d_{2}\right)$ equals the rate region. In particular, this implies that the separation-based scheme depicted in Fig. 2 is an optimal architecture for this problem. We note that the quadraticGaussian two-encoder source-coding problem is not unique in this respect. Prior work has shown this architecture to be optimal for other important problems as well $[4,6,7,8,10,11,12]$.

In fact, the separation-based architecture achieves the rates

$$
\mathcal{R}_{1}^{\star}\left(d_{1}\right) \cap \mathcal{R}_{2}^{\star}\left(d_{2}\right) \cap \mathcal{R}_{\text {sum }}^{\star}\left(d_{1}, d_{2}\right)
$$

even if the source is not Gaussian. Let $\left\{\check{\mathbf{y}}^{n}(i)\right\}_{i=1}^{n}$ be a sequence of zero-mean i.i.d. random vectors, not necessarily Gaussian, with covariance matrix $\boldsymbol{K}_{y}$. We consider the same source-coding problem as before, but with the alternate source $\check{\mathbf{y}}$ in place of $\mathbf{y}$. Let $\check{\mathcal{R}}^{i}\left(d_{1}, d_{2}\right)$ denote the inner bound obtained from Proposition 1.

Proposition 2. The separation-based architecture achieves the rates

$$
\mathcal{R}_{1}^{\star}\left(d_{1}\right) \cap \mathcal{R}_{2}^{\star}\left(d_{2}\right) \cap \mathcal{R}_{\text {sum }}^{\star}\left(d_{1}, d_{2}\right)
$$

for the source $\check{\mathbf{y}}$. That is, $\check{\mathcal{R}}^{i}\left(d_{1}, d_{2}\right)$ contains this set.

Proof. See Appendix A.

Theorem 1 and Proposition 2 together imply that the separation-based architecture requires higher rates to send a Gaussian source than it does to send any other source with the same covariance. In particular, a Gaussian source has the smallest rate region for a given covariance matrix. This result is a twoencoder extension of the well-known fact that a Gaussian source has the highest rate-distortion function for a given variance [13, Ex. 9.7] (see Lapidoth [14] for a stronger version).

\section{Converse Preliminaries}

Oohama [5] determined the rate region when only one of the two distortion constraints is present

$$
\begin{aligned}
& \mathcal{R}^{\star}\left(d_{1}, 1\right)=\mathcal{R}_{1}^{\star}\left(d_{1}\right) \\
& \mathcal{R}^{\star}\left(1, d_{2}\right)=\mathcal{R}_{2}^{\star}\left(d_{2}\right) .
\end{aligned}
$$

As a consequence of his result, it follows that

$$
\mathcal{R}^{\star}\left(d_{1}, d_{2}\right) \subseteq \mathcal{R}_{1}^{\star}\left(d_{1}\right) \cap \mathcal{R}_{2}^{\star}\left(d_{2}\right) .
$$

This outer bound is tight in a certain special case. Let $\mathcal{D}_{G}$ denote the set of matrices $\boldsymbol{D}$ such that

$$
\boldsymbol{D}^{-1}=\boldsymbol{K}_{y}^{-1}+\boldsymbol{\Lambda}
$$


for some diagonal and positive semidefinite matrix $\boldsymbol{\Lambda}$. There is a one-to-one correspondence between $\mathcal{D}_{G}$ and the set of distributed Gaussian test channels. Specifically, $\boldsymbol{D}$ is the covariance matrix of $\mathbf{y}-E[\mathbf{y} \mid \mathbf{u}]$, where $\mathbf{u}$ is a distributed Gaussian test channel with

$$
c_{j}^{2}=\frac{\lambda_{j}}{1+\lambda_{j}} \quad j \in\{1,2\}
$$

and $\lambda_{1}$ and $\lambda_{2}$ are defined by

$$
\boldsymbol{\Lambda}=\left[\begin{array}{cc}
\lambda_{1} & 0 \\
0 & \lambda_{2}
\end{array}\right]
$$

As such, we will sometimes refer to $\boldsymbol{D}$, or equivalently $\boldsymbol{\Lambda}$, as a (distributed Gaussian) test channel. Note that the mutual information between $\mathbf{y}$ and $\mathbf{u}$ can be expressed in terms of $\boldsymbol{D}$

$$
\begin{aligned}
I(\mathbf{y} ; \mathbf{u}) & =h(\mathbf{y})-h(\mathbf{y} \mid \mathbf{u}) \\
& =\frac{1}{2} \log \left((2 \pi e)^{2}\left|\boldsymbol{K}_{y}\right|\right)-\frac{1}{2} \log \left((2 \pi e)^{2}|\boldsymbol{D}|\right) \\
& =\frac{1}{2} \log \frac{\left|\boldsymbol{K}_{y}\right|}{|\boldsymbol{D}|} .
\end{aligned}
$$

Let $\operatorname{diag}\left(\mathcal{D}_{G}\right)$ denote the set of distortion pairs $\left(d_{1}, d_{2}\right)$ such that there exists a $\boldsymbol{D}$ in $\mathcal{D}_{G}$ with top-left entry $d_{1}$ and bottom-right entry $d_{2}$. It is straightforward to verify that $\left(d_{1}, d_{2}\right)$ is in $\operatorname{diag}\left(\mathcal{D}_{G}\right)$ if and only if

$$
\max \left(d_{1}, d_{2}\right) \leq \min \left(1, \rho^{2} \cdot \min \left(d_{1}, d_{2}\right)+1-\rho^{2}\right) .
$$

The set $\operatorname{diag}\left(\mathcal{D}_{G}\right)$ is significant because if $\left(d_{1}, d_{2}\right)$ is not in $\operatorname{diag}\left(\mathcal{D}_{G}\right)$, then the rate region can be determined using existing results.

Lemma 2. If $\left(d_{1}, d_{2}\right)$ is not in $\operatorname{diag}\left(\mathcal{D}_{G}\right)$, then

$$
\mathcal{R}_{1}^{\star}\left(d_{1}\right) \cap \mathcal{R}_{2}^{\star}\left(d_{2}\right) \subseteq \mathcal{R}_{\text {sum }}^{\star}\left(d_{1}, d_{2}\right) .
$$

In particular, the rate region equals

$$
\mathcal{R}^{\star}\left(d_{1}, d_{2}\right)=\mathcal{R}_{1}^{\star}\left(d_{1}\right) \cap \mathcal{R}_{2}^{\star}\left(d_{2}\right) \cap \mathcal{R}_{\text {sum }}^{\star}\left(d_{1}, d_{2}\right) .
$$

The proof is given in Appendix B. In light of this lemma, Lemma 1, and $(5)$, it suffices to show that when $\left(d_{1}, d_{2}\right)$ is in $\operatorname{diag}\left(\mathcal{D}_{G}\right)$,

$$
\mathcal{R}^{\star}\left(d_{1}, d_{2}\right) \subseteq \mathcal{R}_{\text {sum }}^{\star}\left(d_{1}, d_{2}\right)
$$

We show this in the next section.

Our proof uses a characterization of the sum rate of the quadratic Gaussian CEO problem. In the two-encoder version of this problem, encoders 1 and 2 
observe $y_{1}$ and $y_{2}$, respectively, and then communicate with a single decoder as in the original problem. But now $y_{1}$ and $y_{2}$ are of the form

$$
\begin{aligned}
& y_{1}=a_{1} x+n_{1} \\
& y_{2}=a_{2} x+n_{2},
\end{aligned}
$$

where $x, n_{1}$, and $n_{2}$ are independent and Gaussian, and the decoder estimates $x$ instead of $y_{1}$ and $y_{2}$. The distortion measure is again the average squared error. This problem's rate region was determined independently by Oohama [8] and Prabhakaran, Tse, and Ramchandran $[10]^{3}$. Their result shows that the separation-based architecture is optimal for this problem.

For our purpose, we will find it more convenient to consider the problem in which the decoder attempts to estimate $\boldsymbol{\mu}^{T} \mathbf{y}$ for some given vector $\boldsymbol{\mu}$. We call this problem the $\boldsymbol{\mu}$-sum problem. For some values of $\boldsymbol{\mu}$, the $\boldsymbol{\mu}$-sum problem can be coupled to a CEO problem. For these values of $\boldsymbol{\mu}$, it follows that the separation-based architecture is optimal.

Lemma 3. The sum rate for the $\boldsymbol{\mu}$-sum problem with $\mu_{1} \cdot \mu_{2} \geq 0$ and allowable distortion d equals

$$
\inf \left\{\frac{1}{2} \log \frac{\left|\boldsymbol{K}_{y}\right|}{|\boldsymbol{D}|}: \boldsymbol{D} \in \mathcal{D}_{G} \text { and } \boldsymbol{\mu}^{T} \boldsymbol{D} \boldsymbol{\mu} \leq d\right\} .
$$

In Appendix C, we prove an extended version of this lemma that includes a description of the entire rate region. Here we note some properties of $\mathcal{D}_{G}$ and the optimization problem (8). Recall that $\boldsymbol{D}$ is in $\mathcal{D}_{G}$ if there exists a diagonal and positive semidefinite matrix $\boldsymbol{\Lambda}$ such that

$$
\boldsymbol{D}^{-1}=\boldsymbol{K}_{y}^{-1}+\boldsymbol{\Lambda} .
$$

This formula provides a convenient way of evaluating the off-diagonal entry of $\boldsymbol{D}$ in terms of its diagonal entries and $\rho$. Let us write

$$
\boldsymbol{D}=\left[\begin{array}{cc}
d_{1} & \theta \sqrt{d_{1} d_{2}} \\
\theta \sqrt{d_{1} d_{2}} & d_{2}
\end{array}\right]
$$

where $\theta \in(-1,1)$. Equating the off-diagonal entries in (9) gives

$$
\frac{\theta}{\left(1-\theta^{2}\right) \sqrt{d_{1} d_{2}}}=\frac{\rho}{1-\rho^{2}} .
$$

Since $\theta^{2}<1$, it follows that $\theta$ must be positive. But this quadratic equation in $\theta$ has only one positive root

$$
\theta=\frac{\sqrt{\left(1-\rho^{2}\right)^{2}+4 \rho^{2} d_{1} d_{2}}-\left(1-\rho^{2}\right)}{2 \rho \sqrt{d_{1} d_{2}}} .
$$

\footnotetext{
${ }^{3}$ In fact, both works solved the problem for an arbitrary number of encoders, but this generality is not needed at this point.
} 
Thus there is no other matrix in $\mathcal{D}_{G}$ with top-left entry $d_{1}$ and bottom-right entry $d_{2}$. Using (10), the determinant of $\boldsymbol{D}$ can be expressed in terms of $d_{1}$ and $d_{2}$

$$
|\boldsymbol{D}|=\frac{2 d_{1} d_{2}}{\beta\left(d_{1}, d_{2}\right)},
$$

where $\beta(\cdot, \cdot)$ was defined in Section 2. The effect of the product $d_{1} d_{2}$ on $\theta$ is shown in Fig. 4. As $d_{1} d_{2}$ tends to $1, \theta$ converges to $\rho$ and $\boldsymbol{D}$ converges to $\boldsymbol{K}_{y}$. On the other hand, as $d_{1} d_{2}$ tends to zero, $\theta$ also converges to zero, i.e., the errors become asymptotically uncorrelated.

Next we show that every matrix in $\mathcal{D}_{G}$ solves a $\boldsymbol{\mu}$-sum problem for some $\boldsymbol{\mu}$ with $\mu_{1} \cdot \mu_{2}>0$. This fact will be used in the proof of our main result.

Lemma 4. Let

$$
\boldsymbol{D}^{*}=\left[\begin{array}{cc}
d_{1} & \theta^{*} \sqrt{d_{1} d_{2}} \\
\theta^{*} \sqrt{d_{1} d_{2}} & d_{2}
\end{array}\right]
$$

be in $\mathcal{D}_{G}$, and let

$$
\boldsymbol{\mu}^{*}=\left[\begin{array}{l}
\sqrt{d_{2}} \\
\sqrt{d_{1}}
\end{array}\right] .
$$

Then $\boldsymbol{D}^{*}$ is sum-rate optimal for the $\boldsymbol{\mu}^{*}$-sum problem, i.e.,

$$
\frac{1}{2} \log \frac{\left|\boldsymbol{K}_{y}\right|}{\left|\boldsymbol{D}^{*}\right|}=\inf \left\{\frac{1}{2} \log \frac{\left|\boldsymbol{K}_{y}\right|}{|\boldsymbol{D}|}: \boldsymbol{D} \in \mathcal{D}_{G} \text { and } \boldsymbol{\mu}^{* T} \boldsymbol{D} \boldsymbol{\mu}^{*} \leq \boldsymbol{\mu}^{* T} \boldsymbol{D}^{*} \boldsymbol{\mu}^{*}\right\} .
$$

The proof is deferred to Appendix D. It is helpful to note that if the diagonal entries of $\boldsymbol{D}^{*}$ are equal, then the coordinates of $\boldsymbol{\mu}^{*}$ will also be equal. This fact makes the proofs that follow somewhat simpler when the two distortion constraints, $d_{1}$ and $d_{2}$, are equal. As such, the reader is encouraged to keep this case in mind as we turn to the proof of the main result.

\section{Proof of the Main Result}

Recall that we may restrict attention to the case in which $\left(d_{1}, d_{2}\right)$ is in $\operatorname{diag}\left(\mathcal{D}_{G}\right)$. Let us now fix one such distortion pair; we will suppress dependence on $\left(d_{1}, d_{2}\right)$ in what follows. Let $\boldsymbol{D}^{*}$ denote the element of $\mathcal{D}_{G}$ whose top-left and bottomright entries are $d_{1}$ and $d_{2}$, respectively.

Definition 2. For $\theta \in(-1,1)$, let

$$
\boldsymbol{D}_{\theta}=\left[\begin{array}{cc}
d_{1} & \theta \sqrt{d_{1} d_{2}} \\
\theta \sqrt{d_{1} d_{2}} & d_{2}
\end{array}\right]
$$

and define

$$
R_{\text {coop }}(\theta)=\frac{1}{2} \log ^{+} \frac{\left|\boldsymbol{K}_{y}\right|}{\left|\boldsymbol{D}_{\theta}\right|}=\frac{1}{2} \log ^{+} \frac{1-\rho^{2}}{\left(1-\theta^{2}\right) d_{1} d_{2}} .
$$

Let $\boldsymbol{\mu}^{*}$ be the vector defined in (12). Then let

$$
R_{\text {sum }}(\theta)=\inf \left\{\frac{1}{2} \log \frac{\left|\boldsymbol{K}_{y}\right|}{|\boldsymbol{D}|}: \boldsymbol{D} \in \mathcal{D}_{G} \text { and } \boldsymbol{\mu}^{* T} \boldsymbol{D} \boldsymbol{\mu}^{*} \leq \boldsymbol{\mu}^{* T} \boldsymbol{D}_{\theta} \boldsymbol{\mu}^{*}\right\} .
$$


The next lemma is central to the proof of our main result.

Lemma 5. If $\left(R_{1}, R_{2}, d_{1}, d_{2}\right)$ is strict-sense achievable, then

$$
R_{1}+R_{2} \geq \inf _{\theta \in(-1,1)} \max \left(R_{\text {coop }}(\theta), R_{\text {sum }}(\theta)\right) .
$$

Proof. By hypothesis there exists a code $\left(f_{1}^{(n)}, f_{2}^{(n)}, \varphi_{1}^{(n)}, \varphi_{2}^{(n)}\right)$ satisfying (1). Then

$$
\begin{aligned}
n\left(R_{1}+R_{2}\right) & \geq H\left(f_{1}^{(n)}\left(y_{1}^{n}\right), f_{2}^{(n)}\left(y_{2}^{n}\right)\right) \\
& =I\left(\mathbf{y}^{n} ; f_{1}^{(n)}\left(y_{1}^{n}\right), f_{2}^{(n)}\left(y_{2}^{n}\right)\right) \\
& =h\left(\mathbf{y}^{n}\right)-h\left(\mathbf{y}^{n} \mid f_{1}^{(n)}\left(y_{1}^{n}\right), f_{2}^{(n)}\left(y_{2}^{n}\right)\right),
\end{aligned}
$$

where $h(\cdot)$ denotes differential entropy. But

$$
h\left(\mathbf{y}^{n}\right)=\frac{n}{2} \log \left[(2 \pi e)^{2}\left|\boldsymbol{K}_{y}\right|\right]
$$

and

$$
\begin{aligned}
h\left(\mathbf{y}^{n} \mid f_{1}^{(n)}\left(y_{1}^{n}\right), f_{2}^{(n)}\left(y_{2}^{n}\right)\right) & =\sum_{i=1}^{n} h\left(\mathbf{y}^{n}(i) \mid f_{1}^{(n)}\left(y_{1}^{n}\right), f_{2}^{(n)}\left(y_{2}^{n}\right), \mathbf{y}^{n}(1: i-1)\right) \\
& \leq \sum_{i=1}^{n} h\left(\mathbf{y}^{n}(i)-\hat{\mathbf{y}}^{n}(i) \mid f_{1}^{(n)}\left(y_{1}^{n}\right), f_{2}^{(n)}\left(y_{2}^{n}\right)\right) \\
& \leq \sum_{i=1}^{n} h\left(\mathbf{y}^{n}(i)-\hat{\mathbf{y}}^{n}(i)\right),
\end{aligned}
$$

since conditioning reduces entropy. Let $\hat{\boldsymbol{D}}_{i}$ denote the covariance matrix of $\mathbf{y}^{n}(i)-\hat{\mathbf{y}}^{n}(i)$

$$
\hat{\boldsymbol{D}}_{i}=E\left[\left(\mathbf{y}^{n}(i)-\hat{\mathbf{y}}^{n}(i)\right)\left(\mathbf{y}^{n}(i)-\hat{\mathbf{y}}^{n}(i)\right)^{T}\right],
$$

and let

$$
\hat{\boldsymbol{D}}=\frac{1}{n} \sum_{i=1}^{n} \hat{\boldsymbol{D}}_{i}
$$

denote the error covariance matrix of the code. We may assume that $\varphi_{1}^{(n)}$ and $\varphi_{2}^{(n)}$ are MMSE estimators, in which case Theorem 9.6.5 in Cover and Thomas [15] implies that

$$
h\left(\mathbf{y}^{n}(i)-\hat{\mathbf{y}}^{n}(i)\right) \leq \frac{1}{2} \log \left[(2 \pi e)^{2}\left|\hat{\boldsymbol{D}}_{i}\right|\right] .
$$


Applying the concavity of log-det [15, Theorem 16.8.1], we have

$$
\begin{aligned}
\frac{1}{n} h\left(\mathbf{y}^{n} \mid f_{1}^{(n)}\left(y_{1}^{n}\right), f_{2}^{(n)}\left(y_{2}^{n}\right)\right) & \leq \frac{1}{n} \sum_{i=1}^{n} \frac{1}{2} \log \left[(2 \pi e)^{2}\left|\hat{\boldsymbol{D}}_{i}\right|\right] \\
& \leq \frac{1}{2} \log \left[(2 \pi e)^{2}|\hat{\boldsymbol{D}}|\right] .
\end{aligned}
$$

Combining this inequality with (14) and (15) gives

$$
R_{1}+R_{2} \geq \frac{1}{2} \log ^{+} \frac{\left|\boldsymbol{K}_{y}\right|}{|\hat{\boldsymbol{D}}|}
$$

Now

$$
h\left(\mathbf{y}^{n} \mid f_{1}^{(n)}\left(y_{1}^{n}\right), f_{2}^{(n)}\left(y_{2}^{n}\right)\right)>-\infty
$$

by (14). Thus $\hat{\boldsymbol{D}}$ must be nonsingular and hence positive definite. Let us write it as

$$
\hat{\boldsymbol{D}}=\left[\begin{array}{cc}
\hat{d}_{1} & \hat{\theta} \sqrt{\hat{d_{1} \hat{d}_{2}}} \\
\hat{\theta} \sqrt{\hat{d}_{1} \hat{d}_{2}} & \hat{d}_{2}
\end{array}\right],
$$

where $\hat{d}_{1} \leq d_{1}, \hat{d}_{2} \leq d_{2}$, and $\hat{\theta}$ is in $(-1,1)$. Define

$$
\phi=\frac{\hat{\theta} \sqrt{\hat{d}_{1} \hat{d}_{2}}}{\sqrt{d_{1} d_{2}}}
$$

and note that $\phi$ is in $(-1,1)$. Then

$$
\boldsymbol{D}_{\phi}-\hat{\boldsymbol{D}}=\left[\begin{array}{cc}
d_{1}-\hat{d}_{1} & 0 \\
0 & d_{2}-\hat{d}_{2}
\end{array}\right] .
$$

Since $\hat{d}_{1} \leq d_{1}$ and $\hat{d}_{2} \leq d_{2}$, it follows that $\boldsymbol{D}_{\phi}-\hat{\boldsymbol{D}}$ is positive semidefinite, i.e., $\hat{\boldsymbol{D}} \preceq \boldsymbol{D}_{\phi}$. In particular, $|\hat{\boldsymbol{D}}| \leq\left|\boldsymbol{D}_{\phi}\right|[16$, Corollary 7.7.4]. This implies

$$
R_{1}+R_{2} \geq \frac{1}{2} \log ^{+} \frac{\left|\boldsymbol{K}_{y}\right|}{\left|\boldsymbol{D}_{\phi}\right|}=R_{\mathrm{coop}}(\phi)
$$

Next observe that

$$
E\left[\left(\boldsymbol{\mu}^{* T} \mathbf{y}^{n}(i)-\boldsymbol{\mu}^{* T} \hat{\mathbf{y}}^{n}(i)\right)^{2}\right]=\boldsymbol{\mu}^{* T} \hat{\boldsymbol{D}}_{i} \boldsymbol{\mu}^{*} .
$$

In particular,

$$
\frac{1}{n} \sum_{i=1}^{n} E\left[\left(\boldsymbol{\mu}^{* T} \mathbf{y}^{n}(i)-\boldsymbol{\mu}^{* T} \hat{\mathbf{y}}^{n}(i)\right)^{2}\right]=\boldsymbol{\mu}^{* T} \hat{\boldsymbol{D}} \boldsymbol{\mu}^{*} \leq \boldsymbol{\mu}^{* T} \boldsymbol{D}_{\phi} \boldsymbol{\mu}^{*}
$$

i.e., this code achieves distortion $\boldsymbol{\mu}^{* T} \boldsymbol{D}_{\phi} \boldsymbol{\mu}^{*}$ for the $\boldsymbol{\mu}^{*}$-sum problem. Lemma 3 then implies that

$$
R_{1}+R_{2} \geq R_{\text {sum }}(\phi) \text {. }
$$


Combining this with (17) gives

$$
R_{1}+R_{2} \geq \max \left(R_{\text {coop }}(\phi), R_{\text {sum }}(\phi)\right) .
$$

The conclusion follows by taking the infimum over $\phi$ in $(-1,1)$.

The next step is to evaluate the infimum in (13). Examples of $R_{\text {coop }}(\cdot)$ and $R_{\text {sum }}(\cdot)$ are shown in Fig. 5 . We show that these two functions always intersect at the correlation coefficient of $\boldsymbol{D}^{*}$, and at this point, they equal the min-max.

\section{Lemma 6.}

$$
\begin{aligned}
\inf _{\theta \in(-1,1)} \max \left(R_{\text {coop }}(\theta), R_{\text {sum }}(\theta)\right) & =R_{\text {coop }}\left(\theta^{*}\right) \\
& =R_{\text {sum }}\left(\theta^{*}\right) \\
& =\frac{1}{2} \log \frac{\left|\boldsymbol{K}_{y}\right|}{\left|\boldsymbol{D}^{*}\right|} \\
& =\frac{1}{2} \log ^{+}\left[\frac{\left(1-\rho^{2}\right) \beta\left(d_{1}, d_{2}\right)}{2 d_{1} d_{2}}\right] .
\end{aligned}
$$

Proof. Let us write $\boldsymbol{D}^{*}$, the matrix in $\mathcal{D}_{G}$ with diagonal entries $\left(d_{1}, d_{2}\right)$, as

$$
\boldsymbol{D}^{*}=\left[\begin{array}{cc}
d_{1} & \theta^{*} \sqrt{d_{1} d_{2}} \\
\theta^{*} \sqrt{d_{1} d_{2}} & d_{2}
\end{array}\right] .
$$

Then observe that since $\theta^{*}>0$, if $\theta \geq \theta^{*}$, we have

$$
\max \left(R_{\text {coop }}(\theta), R_{\text {sum }}(\theta)\right) \geq R_{\text {coop }}(\theta) \geq R_{\text {coop }}\left(\theta^{*}\right)=\frac{1}{2} \log \frac{\left|\boldsymbol{K}_{y}\right|}{\left|\boldsymbol{D}^{*}\right|} .
$$

On the other hand, if $\theta \leq \theta^{*}$, then since $R_{\text {sum }}(\cdot)$ is nonincreasing,

$$
\max \left(R_{\text {coop }}(\theta), R_{\text {sum }}(\theta)\right) \geq R_{\text {sum }}(\theta) \geq R_{\text {sum }}\left(\theta^{*}\right)=\frac{1}{2} \log \frac{\left|\boldsymbol{K}_{y}\right|}{\left|\boldsymbol{D}^{*}\right|},
$$

where we have used the fact that $\boldsymbol{D}^{*}$ solves the $\boldsymbol{\mu}^{*}$-sum problem. It follows that

$$
\inf _{\theta \in(-1,1)} \max \left(R_{\text {coop }}(\theta), R_{\text {sum }}(\theta)\right)=R_{\text {coop }}\left(\theta^{*}\right)=R_{\text {sum }}\left(\theta^{*}\right)=\frac{1}{2} \log \frac{\left|\boldsymbol{K}_{y}\right|}{\left|\boldsymbol{D}^{*}\right|} .
$$

We conclude the proof by invoking the formula for the determinant of a matrix in $\mathcal{D}_{G}(11)$.

Proof of Theorem 1. As discussed in Section 4, it suffices to show that

$$
\mathcal{R}^{\star}\left(d_{1}, d_{2}\right) \subseteq \mathcal{R}_{\text {sum }}^{\star}\left(d_{1}, d_{2}\right) .
$$

Lemmas 5 and 6 together imply that if the rate-distortion vector $\left(R_{1}, R_{2}, d_{1}, d_{2}\right)$ is strict-sense achievable and $\left(d_{1}, d_{2}\right)$ is $\operatorname{in} \operatorname{diag}\left(\mathcal{D}_{G}\right)$, then

$$
R_{1}+R_{2} \geq \frac{1}{2} \log ^{+}\left[\frac{\left(1-\rho^{2}\right) \beta\left(d_{1}, d_{2}\right)}{2 d_{1} d_{2}}\right] .
$$


On the other hand, Lemma 2 implies this inequality if $\left(R_{1}, R_{2}, d_{1}, d_{2}\right)$ is strictsense achievable and $\left(d_{1}, d_{2}\right)$ in not in $\operatorname{diag}\left(\mathcal{D}_{G}\right)$. It follows that (18) holds whenever $\left(R_{1}, R_{2}, d_{1}, d_{2}\right)$ is strict-sense achievable. Since the right-hand side is continuous in $\left(d_{1}, d_{2}\right)$, this implies that if the point $\left(R_{1}, R_{2}, d_{1}, d_{2}\right)$ is in $\overline{\mathcal{R} \mathcal{D}^{\star}}$, then (18) again holds. This implies the desired conclusion.

\subsection{Reprise}

The argument used in the converse proof can be summarized as follows. Since the distortion constraints only constrain the magnitude of the individual errors, and not their correlation, we view the determination of the sum-rate as an implicit minimization over all possible error covariance matrices, subject to upper bounds on the diagonal elements. We then lower bound the sum rate for each possible error covariance matrix using two approaches. First, we consider the rate needed by a centralized encoder to achieve the given error covariance matrix. Second, we use the existing characterization of the rate region for the CEO problem to solve the $\boldsymbol{\mu}$-sum problem for some $\boldsymbol{\mu}$ vectors. This solution is then used to lower bound the sum rate of the problem under study for a given error covariance matrix. The first bound is most effective when the correlation between the errors is large. The second bound is most effective when the correlation is small. We therefore form a composite bound by taking the maximum of these two lower bounds. The argument is illustrated in Figs. 5 and 6 . Note that both of the lower bounds are needed.

\section{The $M$-sums Problem}

Consider next a generalization of the classical problem in which the decoder attempts to estimate $\boldsymbol{\mu}_{j}^{T} \mathbf{y}$ for a given set of vectors $\boldsymbol{\mu}_{1}, \ldots, \boldsymbol{\mu}_{J}$. We may assume without loss of generality that these vectors are distinct and have unit norm.

Define the matrix

$$
\boldsymbol{M}=\left[\boldsymbol{\mu}_{1} \boldsymbol{\mu}_{2} \cdots \boldsymbol{\mu}_{J}\right]
$$

consisting of the column vectors $\boldsymbol{\mu}_{1}, \ldots, \boldsymbol{\mu}_{J}$ side-by-side. The problem is then to reproduce the vector $\boldsymbol{M}^{T} \mathbf{y}$ subject to separate constraints on the average squared error of each component. We call this the $\boldsymbol{M}$-sums problem. Note that the classical quadratic Gaussian two-encoder source coding problem can be viewed as an instance of the $\boldsymbol{M}$-sums problem with $\boldsymbol{M}$ equal to the identity matrix. We will show that the techniques used to solve that problem can be used to solve the general $\boldsymbol{M}$-sums problem if the vectors $\boldsymbol{\mu}_{1}, \ldots \boldsymbol{\mu}_{J}$ satisfy a certain condition. Specifically, we will require that the product of the two coordinates of each vector is nonnegative

$$
\boldsymbol{\mu}_{j 1} \cdot \boldsymbol{\mu}_{j 2} \geq 0 \quad \forall j \in\{1, \ldots, J\} .
$$

This condition is satisfied if and only for each $j$ either both coordinates of $\boldsymbol{\mu}_{j}$ are nonnegative or both are nonpositive. From a source-coding perspective, these 
two cases are essentially equivalent, so for simplicity we will assume that the components of $\boldsymbol{\mu}_{j}$ are nonnegative for each $j$.

The condition in (19) depends on our standing assumption that $0<\rho<1$. If $\rho$ is negative, then the condition in (19) becomes

$$
\boldsymbol{\mu}_{j 1} \cdot \boldsymbol{\mu}_{j 2} \leq 0 \quad \forall j \in\{1, \ldots, J\}
$$

Note that either way, the condition includes the case when $\boldsymbol{M}$ is the identity matrix, i.e., the classical version of the problem.

\subsection{Main Result}

In this section, we use $\mathcal{R}_{j}^{\star}\left(d_{j}\right)$ to denote the rate region of the $\boldsymbol{\mu}_{j}$-sum problem with distortion constraint $d_{j}$. Let $R_{\text {sum }}^{\star}\left(d_{1}, \ldots, d_{J}\right)$ denote the minimum sum rate for the $M$-sums problem achieved by the separation-based scheme

$$
\begin{aligned}
& R_{\text {sum }}^{\star}\left(d_{1}, \ldots, d_{J}\right) \\
& \quad=\inf \left\{\frac{1}{2} \log \frac{\left|\boldsymbol{K}_{y}\right|}{|\boldsymbol{D}|}: \boldsymbol{D} \in \mathcal{D}_{G} \text { and } \boldsymbol{\mu}_{j}^{T} \boldsymbol{D} \boldsymbol{\mu}_{j} \leq d_{j} \forall j \in\{1, \ldots, J\}\right\} .
\end{aligned}
$$

Then let $\mathcal{R}_{\text {sum }}^{\star}\left(d_{1}, \ldots, d_{J}\right)$ denote the set of rate pairs whose sum is at least $R_{\text {sum }}^{\star}\left(d_{1}, \ldots, d_{J}\right)$

$$
\mathcal{R}_{\text {sum }}^{\star}\left(d_{1}, \ldots, d_{J}\right)=\left\{\left(R_{1}, R_{2}\right): R_{1}+R_{2} \geq R_{\text {sum }}^{\star}\left(d_{1}, \ldots, d_{J}\right)\right\} .
$$

In terms of these sets, the separation-based architecture achieves the following inner bound.

Lemma 7. For the $\boldsymbol{M}$-sums problem, the separation-based architecture achieves the rates

$$
\mathcal{R}_{\text {sum }}^{\star}\left(d_{1}, \ldots, d_{J}\right) \cap \bigcap_{j=1}^{J} \mathcal{R}_{j}^{\star}\left(d_{j}\right) .
$$

The proof is elementary but somewhat involved and is given in Appendix E. The main result of this section is the following theorem that shows that this inner bound equals the rate region.

Theorem 2. The rate region of the $\boldsymbol{M}$-sums problem equals

$$
\mathcal{R}_{\text {sum }}^{\star}\left(d_{1}, \ldots, d_{J}\right) \cap \bigcap_{j=1}^{J} \mathcal{R}_{j}^{\star}\left(d_{j}\right) .
$$

The proof parallels that of Theorem 1. In particular, we use functions similar to $R_{\text {coop }}(\cdot)$ and $R_{\text {sum }}(\cdot)$. The details are given in Appendix F.

By mimicking the proof of Proposition 2, one can show that the separationbased architecture achieves the rates in (21) even if the source is not Gaussian. Theorem 2 then implies that, as with the classical version of the problem, the separation-based inner bound and the rate region are both smallest for a Gaussian source. 


\subsection{The Remote-source Problem}

As an application of Theorem 2, consider the remote-source version of the original problem. Here the encoders' observations are viewed as an underlying source, $\tilde{\mathbf{y}}$, plus additive noise

$$
\begin{aligned}
& y_{1}=\tilde{y}_{1}+n_{1} \\
& y_{2}=\tilde{y}_{2}+n_{2}
\end{aligned}
$$

where $\tilde{\mathbf{y}}, n_{1}$, and $n_{2}$ are independent and Gaussian. We assume these random variables have zero mean and

$$
\begin{aligned}
E\left[\tilde{y}_{1}^{2}\right] & =\sigma_{1}^{2} \leq 1 \\
E\left[\tilde{y}_{2}^{2}\right] & =\sigma_{2}^{2} \leq 1 \\
E\left[\tilde{y}_{1} \tilde{y}_{2}\right] & =\rho \\
E\left[n_{1}^{2}\right] & =1-\sigma_{1}^{2} \\
E\left[n_{2}^{2}\right] & =1-\sigma_{2}^{2}
\end{aligned}
$$

so that $\mathbf{y}$ has covariance matrix $\boldsymbol{K}_{y}$. The aim is to reproduce $\tilde{y}_{1}$ and $\tilde{y}_{2}$ subject to distortion constraints $d_{1}$ and $d_{2}$, respectively. A partial characterization of the rate region for this problem was obtained by Oohama [17]. By coupling this problem to an $\boldsymbol{M}$-sums problem, we can determine the rate region completely.

Corollary 1. The rate region for the remote-source problem with distortion constraints $d_{1}$ and $d_{2}$ equals the rate region for the $\boldsymbol{M}$-sums problem with distortion constraints $d_{1}-\gamma_{1}$ and $d_{2}-\gamma_{2}$, where

$$
\boldsymbol{M}=\frac{1}{1-\rho^{2}}\left[\begin{array}{cc}
\sigma_{1}^{2}-\rho^{2} & \rho\left(1-\sigma_{2}^{2}\right) \\
\rho\left(1-\sigma_{1}^{2}\right) & \sigma_{2}^{2}-\rho^{2}
\end{array}\right]=\left[\begin{array}{ll}
\boldsymbol{\mu}_{1} & \boldsymbol{\mu}_{2}
\end{array}\right]
$$

and

$$
\begin{aligned}
& \gamma_{1}=\frac{\left(\sigma_{1}^{2}-\rho^{2}\right)\left(1-\sigma_{1}^{2}\right)}{1-\rho^{2}} \\
& \gamma_{2}=\frac{\left(\sigma_{2}^{2}-\rho^{2}\right)\left(1-\sigma_{2}^{2}\right)}{1-\rho^{2}}
\end{aligned}
$$

Proof. Standard calculations show that $E\left[\tilde{y}_{1} \mid \mathbf{y}\right]=\boldsymbol{\mu}_{1}^{T} \mathbf{y}$, and in particular, $\tilde{y}_{1}$ can be written

$$
\tilde{y}_{1}=\boldsymbol{\mu}_{1}^{T} \mathbf{y}+\tilde{n}_{1},
$$

where $\tilde{n}_{1}$ is Gaussian, independent of $\mathbf{y}$, and has mean zero and variance $\gamma_{1}$. Then for any random variable $u$ such that $\tilde{\mathbf{y}} \leftrightarrow \mathbf{y} \leftrightarrow u$, we have

$$
\begin{aligned}
E\left[\left(\tilde{y}_{1}-E\left[\tilde{y}_{1} \mid u\right]\right)^{2}\right] & =E\left[\left(\tilde{y}_{1}-\boldsymbol{\mu}_{1}^{T} \mathbf{y}+\boldsymbol{\mu}_{1}^{T} \mathbf{y}-E\left[\tilde{y}_{1} \mid u\right]\right)^{2}\right] \\
& =E\left[\left(\tilde{n}_{1}+\boldsymbol{\mu}_{1}^{T} \mathbf{y}-E\left[\boldsymbol{\mu}_{1}^{T} \mathbf{y} \mid u\right]\right)^{2}\right] \\
& =\gamma_{1}+E\left[\left(\boldsymbol{\mu}_{1}^{T} \mathbf{y}-E\left[\boldsymbol{\mu}_{1}^{T} \mathbf{y} \mid u\right]\right)^{2}\right]
\end{aligned}
$$


Now consider a pair of encoders, $f_{1}^{(n)}$ and $f_{2}^{(n)}$. Since the source is i.i.d., for any time $i$,

$$
\tilde{\mathbf{y}}(i) \leftrightarrow \mathbf{y}(i) \leftrightarrow\left(f_{1}^{(n)}\left(y_{1}^{n}\right), f_{2}^{(n)}\left(y_{2}^{n}\right)\right) .
$$

Thus by (22),

$$
\begin{aligned}
E\left[\left(\tilde{y}_{1}(i)-E\left[\tilde{y}_{1}(i) \mid f_{1}^{(n)}\left(y_{1}^{n}\right), f_{2}^{(n)}\left(y_{2}^{n}\right)\right]\right)^{2}\right] & \\
& =\gamma_{1}+E\left[\left(\boldsymbol{\mu}_{1}^{T} \mathbf{y}(i)-E\left[\boldsymbol{\mu}_{1}^{T} \mathbf{y}(i) \mid f_{1}^{(n)}\left(y_{1}^{n}\right), f_{2}^{(n)}\left(y_{2}^{n}\right)\right]\right)^{2}\right] .
\end{aligned}
$$

By averaging both sides of this equation over time, we see that any encoder that achieves distortion $d_{1}$ for $\tilde{y}_{1}$ must achieve distortion $d_{1}-\gamma_{1}$ for $\boldsymbol{\mu}_{1}^{T} \mathbf{y}$. Likewise, any encoder that achieves distortion $d_{2}$ for $\tilde{y}_{2}$ must achieve distortion $d_{2}-\gamma_{2}$ for $\boldsymbol{\mu}_{2}^{T} \mathbf{y}$. The conclusion follows.

Observe that this proof does not require the assumption that $0<\rho<1$, only that $\rho^{2}<1$. If $0<\rho<1$, then $\boldsymbol{\mu}_{1}$ and $\boldsymbol{\mu}_{2}$ satisfy the condition in (19). On the other hand, if $-1<\rho<0$, then $\boldsymbol{\mu}_{1}$ and $\boldsymbol{\mu}_{2}$ satisfy the condition in (20). Since the cases $\rho=0, \rho=-1$, and $\rho=1$ can be solved using existing techniques, the rate region for the remote source problem is solved for any value of $\rho$.

\section{Many Sources}

Our technique can be used to determine the sum rate for more than two sources if a certain symmetry condition holds. Suppose now that there are $L$ jointly Gaussian sources, $y_{1}, \ldots, y_{L}$, with covariance matrix

$$
\boldsymbol{K}_{y}=\left[\begin{array}{cccc}
1 & \rho & \cdots & \rho \\
\rho & 1 & \cdots & \rho \\
\vdots & \vdots & \ddots & \vdots \\
\rho & \rho & \cdots & 1
\end{array}\right]
$$

for some $0<\rho<1$. That is, the source components are Gaussian, exchangeable, and positively correlated. We assume that the sources are separately encoded, as shown in Fig. 9, and that $L$ distortion constraints are imposed on the individual reproductions

$$
d_{\ell} \geq \frac{1}{n} \sum_{i=1}^{n} E\left[\left(y_{\ell}^{n}(i)-\hat{y}_{\ell}^{n}(i)\right)^{2}\right] \quad \text { for all } \ell \text { in }\{1, \ldots, L\} .
$$

The separation-based scheme yields an inner bound on the rate region, and in particular, an upper bound on the sum rate. As in the case of two sources, let $\mathcal{D}_{G}$ denote the set of matrices $\boldsymbol{D}$ such that

$$
\boldsymbol{D}^{-1}=\boldsymbol{K}_{y}^{-1}+\boldsymbol{\Lambda}
$$


for some diagonal and positive semidefinite matrix $\boldsymbol{\Lambda}$. The sum rate achieved by the separation-based scheme is then

$$
\inf \left\{\frac{1}{2} \log \frac{\left|\boldsymbol{K}_{y}\right|}{|\boldsymbol{D}|}: \boldsymbol{D} \in \mathcal{D}_{G} \text { and } \mathbf{e}_{\ell}^{T} \boldsymbol{D} \mathbf{e}_{\ell} \leq d_{\ell} \forall \ell \in\{1, \ldots, L\}\right\},
$$

where $\mathbf{e}_{\ell}$ denotes the vector with one in position $\ell$ and zero elsewhere. By following the proof of Theorem 1, one can show that this sum rate is optimal if the distortion constraints $d_{1}, \ldots, d_{L}$ are equal.

Theorem 3. If $d_{1}=d_{2}=\cdots=d_{L}=d$, then the separation-based architecture is sum-rate optimal. In particular, the sum rate is given by (23). Furthermore, in this case the infimum in (23) is achieved by a $\boldsymbol{D}$ in $\mathcal{D}_{G}$ of the form

$$
\boldsymbol{D}^{-1}=\boldsymbol{K}_{y}^{-1}+\lambda \boldsymbol{I}
$$

for some $\lambda \geq 0$.

The proof is given in Appendix G. As with the $\boldsymbol{M}$-sums problem, it is possible to mimic Proposition 2 and show that the separation-based architecture achieves the sum rate in (23) even if the source is not Gaussian. It follows that the Gaussian source has the largest sum rate among all exchangeable and positively correlated sources when all of the distortion constraints are equal.

\section{Concluding Remarks}

We determined the rate region of the quadratic Gaussian two-encoder sourcecoding problem. This result implies that a simple architecture that separates the analog and digital aspects of the compression is optimal, and that this architecture requires higher rates to send a Gaussian source than it does to send any other source with the same covariance. We also described how our proof technique can be extended to determine the sum rate of some generalizations of this problem. We now comment on two aspects of our results.

\subsection{An Extremal Result}

One consequence of our main result is that there is no loss of optimality in using Gaussian auxiliary random variables in the separation-based inner bound. More precisely, the regions $\mathcal{R}^{i}\left(d_{1}, d_{2}\right)$ and $\mathcal{R}_{G}^{i}\left(d_{1}, d_{2}\right)$ defined in (2) and (3) are equal. In particular, these two regions have the same sum rate. Thus to the optimization problem

$$
\begin{aligned}
\operatorname{minimize} & I(\mathbf{y} ; \mathbf{u}) \\
\text { subject to } & u_{1} \leftrightarrow y_{1} \leftrightarrow y_{2} \leftrightarrow u_{2} \\
& E\left[\left(y_{j}-E\left[y_{j} \mid \mathbf{u}\right]\right)^{2}\right] \leq d_{j} \quad j \in\{1,2\}
\end{aligned}
$$


we can add the constraint

$$
(\mathbf{y}, \mathbf{u}) \text { is jointly Gaussian }
$$

without changing the optimal value. The same is true, of course, of the optimization problem

$$
\begin{aligned}
\operatorname{maximize} & h(\mathbf{y} \mid \mathbf{u}) \\
\text { subject to } & u_{1} \leftrightarrow y_{1} \leftrightarrow y_{2} \leftrightarrow u_{2} \\
& E\left[\left(y_{j}-E\left[y_{j} \mid \mathbf{u}\right]\right)^{2}\right] \leq d_{j} \quad j \in\{1,2\}
\end{aligned}
$$

This is akin to the well-known fact that the Gaussian distribution maximizes entropy for a given covariance. But this result is more subtle in that the conditional covariance of $\mathbf{y}$ given $\mathbf{u}$ is not fixed, and by using non-Gaussian $\mathbf{u}$, one can potentially realize conditional covariances that are unattainable with Gaussian distributions. Evidently the entropy-maximizing property of the Gaussian distribution more than compensates for its smaller set of achievable conditional covariances.

Using Theorem 2, it is possible to generalize this result to allow distortion constraints on linear combinations of the source variables $y_{1}$ and $y_{2}$. It is also possible to prove a multi-letter version of this result by first proving a multiletter version of the inner bound, in which several source symbols are treated as a single "supersymbol." Whether one can prove any of these extremal results without reference to the source-coding setup that is the subject of this paper is an interesting open question.

\subsection{Source Augmentation}

The most noteworthy aspect of our proof is the random variable $x$ that we add to the source $\mathbf{y}$ in Appendix $\mathrm{C}$ to solve the $\boldsymbol{\mu}$-sum problem. Unlike other more typical auxiliary random variables, $x$ does not represent a component of the code. Rather, it is used to aid the analysis by inducing conditional independence among the observations, which allows us to couple our problem to a CEO problem. Of course, there are many random variables that will induce conditional independence. The role of Lemma 4 is to identify the best one.

This technique of augmenting the source to induce conditional independence has proven useful in other contexts as well. Ozarow [18] used it to prove the converse for the Gaussian two-descriptions problem. Wang and Viswanath [19] used it to determine the sum rate for the Gaussian vector multiple-descriptions problem with individual and central decoders. Wagner and Anantharam $[11,12]$ used it to prove an outer bound for the discrete multiterminal source-coding problem.

Recently, we have generalized the CEO result to sources whose correlation satisfies a certain tree condition [20]. This suggests an approach for generalizing the results in this paper. Specifically, one could potentially augment the source to couple a given distributed source coding problem to this tree problem 
instead of the more restrictive CEO problem. Determining whether this revised approach yields stronger results is a worthwhile question for future research.

\section{Acknowledgment}

We wish to thank Venkat Anantharam and Jun Chen for helpful discussions. We would also like to thank the anonymous reviewers for carefully checking the manuscript and suggesting many improvements.

\section{A Proof of Proposition 2}

Let $\left(R_{1}, R_{2}\right)$ be a rate pair in

$$
\mathcal{R}_{1}^{\star}\left(d_{1}\right) \cap \mathcal{R}_{2}^{\star}\left(d_{2}\right) \cap \mathcal{R}_{\text {sum }}^{\star}\left(d_{1}, d_{2}\right) .
$$

By Lemma 1 , there exists a $\mathbf{u}$ in $\mathcal{U}_{G}\left(d_{1}, d_{2}\right)$ such that

$$
\begin{aligned}
R_{1} & \geq I\left(y_{1} ; u_{1} \mid u_{2}\right) \\
R_{2} & \geq I\left(y_{2} ; u_{2} \mid u_{1}\right) \\
R_{1}+R_{2} & \geq I(\mathbf{y} ; \mathbf{u}) .
\end{aligned}
$$

Now $\mathbf{u}$ can be expressed as

$$
\begin{aligned}
& u_{1}=c_{1} y_{1}+z_{1} \\
& u_{2}=c_{2} y_{2}+z_{2}
\end{aligned}
$$

for some coefficients $c_{1}$ and $c_{2}$ in $[0,1)$, where $z_{1}, z_{2}$, and $\mathbf{y}$ are independent and $\mathbf{z}$ is Gaussian. Now construct auxiliary random variables $\check{u}_{1}$ and $\check{u}_{2}$ for the true source via

$$
\begin{aligned}
& \check{u}_{1}=c_{1} \check{y}_{1}+z_{1} \\
& \check{u}_{2}=c_{2} \check{y}_{2}+z_{2}
\end{aligned}
$$

with $\mathbf{z}$ independent of $\check{\mathbf{y}}$. Note that the Markov condition

$$
\check{u}_{1} \leftrightarrow \check{y}_{1} \leftrightarrow \check{y}_{2} \leftrightarrow \check{u}_{2}
$$

is satisfied and that $(\check{\mathbf{y}}, \check{\mathbf{u}})$ and $(\mathbf{y}, \mathbf{u})$ have the same second-order statistics. Thus the error in the linear minimum mean-squared error estimate of $\check{y}_{j}$ given $\check{\mathbf{u}}, \operatorname{LMMSE}\left(\check{y}_{j} \mid \check{\mathbf{u}}\right)$, equals the error in the linear minimum mean-squared error estimate of $y_{j}$ given $\mathbf{u}$,

$$
\operatorname{LMMSE}\left(\check{y}_{j} \mid \check{\mathbf{u}}\right)=\operatorname{LMMSE}\left(y_{j} \mid \mathbf{u}\right) \quad j \in\{1,2\} .
$$

But

$$
E\left[\left(\check{y}_{j}-E\left[\check{y}_{j} \mid \check{\mathbf{u}}\right]\right)^{2}\right] \leq \operatorname{LMMSE}\left(\check{y}_{j} \mid \check{\mathbf{u}}\right) \quad j \in\{1,2\},
$$


and for jointly Gaussian random variables, the linear minimum mean-squared error estimate is also the conditional expectation. Since $\mathbf{u}$ is in $\mathcal{U}_{G}\left(d_{1}, d_{2}\right)$, this implies

$$
E\left[\left(\check{y}_{j}-E\left[\check{y}_{j} \mid \check{\mathbf{u}}\right]\right)^{2}\right] \leq d_{j} \quad j \in\{1,2\} .
$$

It follows that $\check{\mathbf{u}}$ is in $\mathcal{U}\left(d_{1}, d_{2}\right)$. Next, we show that $\left(R_{1}, R_{2}\right)$ satisfies

$$
\begin{aligned}
R_{1} & \geq I\left(\check{y}_{1} ; \check{u}_{1} \mid \check{u}_{2}\right) \\
R_{2} & \geq I\left(\check{y}_{2} ; \check{u}_{2} \mid \check{u}_{1}\right) \\
R_{1}+R_{2} & \geq I(\check{\mathbf{y}} ; \check{\mathbf{u}}) .
\end{aligned}
$$

To prove this, it suffices to show that

$$
\begin{aligned}
I\left(y_{1} ; u_{1} \mid u_{2}\right) & \geq I\left(\check{y}_{1} ; \check{u}_{1} \mid \check{u}_{2}\right) \\
I\left(y_{2} ; u_{2} \mid u_{1}\right) & \geq I\left(\check{y}_{2} ; \check{u}_{2} \mid \check{u}_{1}\right) \\
I(\mathbf{y} ; \mathbf{u}) & \geq I(\check{\mathbf{y}} ; \check{\mathbf{u}}) .
\end{aligned}
$$

By symmetry, it suffices to prove the last two inequalities. Let

$$
\alpha_{2}=\frac{E\left[u_{1} u_{2}\right]}{E\left[u_{1}^{2}\right]} .
$$

Then we have

$$
\begin{aligned}
I\left(\check{y}_{2} ; \check{u}_{2} \mid \check{u}_{1}\right) & \stackrel{(a)}{=} h\left(\check{u}_{2} \mid \check{u}_{1}\right)-h\left(\check{u}_{2} \mid \check{y}_{2}\right) \\
& \stackrel{(b)}{=} h\left(\check{u}_{2}-\alpha_{2} \check{u}_{1} \mid \check{u}_{1}\right)-h\left(z_{2}\right) \\
& \stackrel{(c)}{\leq} h\left(\check{u}_{2}-\alpha_{2} \check{u}_{1}\right)-h\left(z_{2}\right) \\
& \stackrel{(d)}{\leq} h\left(u_{2}-\alpha_{2} u_{1}\right)-h\left(z_{2}\right) \\
& \stackrel{(e)}{=} I\left(y_{2} ; u_{2} \mid u_{1}\right),
\end{aligned}
$$

where

(a) follows from the Markov condition $\check{u}_{2} \leftrightarrow \check{y}_{2} \leftrightarrow \check{u}_{1}$,

(b) follows because differential entropy is invariant to shifts,

(c) follows because conditioning reduces differential entropy,

(d) follows from the fact that $\mathbf{u}$ has the same covariance as $\check{\mathbf{u}}$, and the Gaussian distribution maximizes differential entropy for a given variance, and

(e) follows because steps $(c)$ and $(d)$ are tight if $(\check{\mathbf{y}}, \check{\mathbf{u}})$ is Gaussian. 
We can prove (24) via similar reasoning

$$
\begin{aligned}
I(\check{\mathbf{y}} ; \check{\mathbf{u}}) & =h(\check{\mathbf{u}})-h(\check{\mathbf{u}} \mid \check{\mathbf{y}}) \\
& =h(\check{\mathbf{u}})-h(\mathbf{z}) \\
& \leq h(\mathbf{u})-h(\mathbf{z}) \\
& =I(\mathbf{y} ; \mathbf{u}) .
\end{aligned}
$$

It follows that the rate pair $\left(R_{1}, R_{2}\right)$ belongs to $\check{\mathcal{R}}^{i}\left(d_{1}, d_{2}\right)$.

\section{B Converse for a Special Case}

Proof of Lemma 2. The conclusion is easily verified if $\min \left(d_{1}, d_{2}\right) \geq 1$, so assume instead that $\min \left(d_{1}, d_{2}\right)<1$. Without loss of generality, let us assume that $d_{1}=\min \left(d_{1}, d_{2}\right)$. Then by $(7)$, we must have

$$
\rho^{2} d_{1}+1-\rho^{2}<d_{2} .
$$

If this holds, then

$$
\mathcal{R}_{\text {sum }}^{\star}\left(d_{1}, \rho^{2} d_{1}+1-\rho^{2}\right) \subseteq \mathcal{R}_{\text {sum }}^{\star}\left(d_{1}, d_{2}\right) .
$$

But one can verify directly that

$$
\mathcal{R}_{\text {sum }}^{\star}\left(d_{1}, \rho^{2} d_{1}+1-\rho^{2}\right)=\left\{\left(R_{1}, R_{2}\right): R_{1}+R_{2} \geq \frac{1}{2} \log \frac{1}{d_{1}}\right\} .
$$

Now via calculus one can show that if $\left(R_{1}, R_{2}\right)$ is in $\mathcal{R}_{1}^{\star}\left(d_{1}\right)$ then $R_{1}$ and $R_{2}$ must satisfy

$$
R_{1}+R_{2} \geq \frac{1}{2} \log \frac{1}{d_{1}} .
$$

It follows that

$$
\mathcal{R}_{1}^{\star}\left(d_{1}\right) \subseteq \mathcal{R}_{\text {sum }}^{\star}\left(d_{1}, d_{2}\right)
$$

In particular, we have

$$
\mathcal{R}_{1}^{\star}\left(d_{1}\right) \cap \mathcal{R}_{2}^{\star}\left(d_{2}\right)=\mathcal{R}_{1}^{\star}\left(d_{1}\right) \cap \mathcal{R}_{2}^{\star}\left(d_{2}\right) \cap \mathcal{R}_{\text {sum }}^{\star}\left(d_{1}, d_{2}\right) .
$$

The result then follows from (5) and Lemma 1.

\section{The $\mu$-sum Problem}

In this appendix, we determine the rate region for the $\boldsymbol{\mu}$-sum problem if $\boldsymbol{\mu}$ satisfies $\mu_{1} \cdot \mu_{2} \geq 0$. If $\mu_{1} \cdot \mu_{2}=0$, then the rate region has already been determined by Oohama [5], so we shall assume that $\mu_{1} \cdot \mu_{2}>0$. 
We begin by noting that if $\boldsymbol{\mu}$ and the allowable distortion are both scaled by the same factor, then the rate region remains unchanged. We may therefore assume that $\boldsymbol{\mu}$ is normalized. In particular, we may assume that

$$
\mu_{1} \cdot \mu_{2}=\frac{\gamma^{2}}{\rho} \cdot \mathrm{SNR}_{1} \cdot \mathrm{SNR}_{2}
$$

where

$$
\begin{aligned}
& \mathrm{SNR}_{1}=\frac{\rho}{1-\rho^{2}}\left(\frac{\mu_{1}}{\mu_{2}}+\rho\right) \\
& \mathrm{SNR}_{2}=\frac{\rho}{1-\rho^{2}}\left(\frac{\mu_{2}}{\mu_{1}}+\rho\right)
\end{aligned}
$$

and

$$
\gamma^{-1}=1+\mathrm{SNR}_{1}+\mathrm{SNR}_{2} .
$$

This normalization is convenient because, as we shall see, it admits a particularly simple coupling to a CEO problem.

Lemma 8. Suppose the vector $\boldsymbol{\mu}$ satisfies $\mu_{1} \cdot \mu_{2}>0$ and the normalization (25). Then the rate region for the $\boldsymbol{\mu}$-sum problem with allowable distortion $d$ equals

$$
\begin{aligned}
\left\{\left(R_{1}, R_{2}\right)\right. & : \text { there exist } r_{1} \geq 0, r_{2} \geq 0 \text { such that } \\
R_{1} & \geq \frac{1}{2} \log ^{+}\left[\frac{1}{d+\gamma}\left(1+\operatorname{SNR}_{2}\left(1-2^{-2 r_{2}}\right)\right)^{-1}\right]+r_{1} \\
R_{2} & \geq \frac{1}{2} \log ^{+}\left[\frac{1}{d+\gamma}\left(1+\operatorname{SNR}_{1}\left(1-2^{-2 r_{1}}\right)\right)^{-1}\right]+r_{2} \\
R_{1}+R_{2} & \geq \frac{1}{2} \log ^{+}\left[\frac{1}{d+\gamma}\right]+r_{1}+r_{2} \\
\frac{1}{d+\gamma} & \left.\leq 1+\sum_{j=1}^{2} \operatorname{SNR}_{j}\left(1-2^{-2 r_{j}}\right)\right\} .
\end{aligned}
$$

In particular, the sum rate equals

$$
\begin{gathered}
\inf \left\{\frac{1}{2} \log ^{+}\left[\frac{1}{d+\gamma}\right]+r_{1}+r_{2}: r_{1} \geq 0, r_{2} \geq 0,\right. \text { and } \\
\left.1+\sum_{j=1}^{2} \operatorname{SNR}_{j}\left(1-2^{-2 r_{j}}\right) \geq \frac{1}{d+\gamma}\right\}
\end{gathered}
$$

or, equivalently, by

$$
\inf \left\{\frac{1}{2} \log \frac{\left|\boldsymbol{K}_{y}\right|}{|\boldsymbol{D}|}: \boldsymbol{D} \in \mathcal{D}_{G} \text { and } \boldsymbol{\mu}^{T} \boldsymbol{D} \boldsymbol{\mu} \leq d\right\} .
$$

Furthermore, the infimum in (31) is achieved by a unique feasible $\boldsymbol{D}$. 
Proof. Let

$$
a_{j}=\left(\frac{\mathrm{SNR}_{j}}{1+\mathrm{SNR}_{j}}\right)^{1 / 2} j \in\{1,2\} .
$$

Clearly $a_{1}<1$ and $a_{2}<1$. Using (26) and (27), one can verify that $a_{1} a_{2}=\rho$. It follows that $a_{1}$ and $a_{2}$ are each contained in $(\rho, 1)$. Let $x, n_{1}$, and $n_{2}$ be independent zero-mean Gaussian random variables with

$$
\begin{aligned}
& E\left[x^{2}\right]=1 \\
& E\left[n_{j}^{2}\right]=1-a_{j}^{2} \quad j \in\{1,2\} .
\end{aligned}
$$

Since $\left(a_{1} x+n_{1}, a_{2} x+n_{2}\right)$ has covariance matrix $\boldsymbol{K}_{y}$, we can couple these variables to $\mathbf{y}$ to create a CEO problem

$$
\begin{aligned}
& y_{1}=a_{1} x+n_{1} \\
& y_{2}=a_{2} x+n_{2} .
\end{aligned}
$$

The SNR notation is justified by the fact that

$$
\mathrm{SNR}_{j}=\frac{a_{j}^{2}}{1-a_{j}^{2}}=\frac{\operatorname{Var}\left(a_{j} x\right)}{\operatorname{Var}\left(n_{j}\right)} \quad j \in\{1,2\} .
$$

Now starting with (25), we have

$$
\mu_{1} \cdot \mu_{2}=\sqrt{\mu_{1} \mu_{2}} \cdot \frac{\gamma}{\sqrt{\rho}} \cdot \sqrt{\mathrm{SNR}_{1} \mathrm{SNR}_{2}} .
$$

Substituting for $\mathrm{SNR}_{1}$ and $\mathrm{SNR}_{2}$ and rearranging gives

$$
\mu_{1}=\gamma \cdot \sqrt{\frac{\rho \mu_{1}+\rho^{2} \mu_{2}}{\mu_{2}+\rho \mu_{1}}} \cdot \frac{\mu_{2}+\rho \mu_{1}}{\left(1-\rho^{2}\right) \mu_{2}} .
$$

But

$$
\frac{\mu_{2}+\rho \mu_{1}}{\left(1-\rho^{2}\right) \mu_{2}}=1+\mathrm{SNR}_{1}=\frac{1}{1-a_{1}^{2}}
$$

and

$$
\sqrt{\frac{\rho \mu_{1}+\rho^{2} \mu_{2}}{\mu_{2}+\rho \mu_{1}}}=a_{1} .
$$

Thus

$$
\begin{aligned}
\mu_{1} & =\gamma \cdot a_{1} \cdot \frac{1}{1-a_{1}^{2}} \\
& =\gamma \cdot \frac{\mathrm{SNR}_{1}}{a_{1}} .
\end{aligned}
$$

Similarly,

$$
\mu_{2}=\gamma \cdot \frac{\mathrm{SNR}_{2}}{a_{2}} .
$$


Now using the fact that $a_{1} a_{2}=\rho$, we have

$$
\begin{aligned}
{\left[\begin{array}{ll}
a_{1} & a_{2}
\end{array}\right] \cdot \boldsymbol{K}_{y}^{-1} } & =\frac{1}{1-\rho^{2}}\left[\begin{array}{ll}
a_{1}-\rho a_{2} & a_{2}-\rho a_{1}
\end{array}\right] \\
& =\frac{1}{1-a_{1}^{2} a_{2}^{2}}\left[\frac{a_{1}^{2}-a_{1}^{2} a_{2}^{2}}{a_{1}} \frac{a_{2}^{2}-a_{1}^{2} a_{2}^{2}}{a_{2}^{2}}\right]
\end{aligned}
$$

Substituting for $a_{1}$ and $a_{2}$ using (32), this gives

$$
\left[\begin{array}{ll}
a_{1} & a_{2}
\end{array}\right] \cdot \boldsymbol{K}_{y}^{-1}=\left[\begin{array}{cc}
\frac{\gamma \mathrm{SNR}_{1}}{a_{1}} & \frac{\gamma \mathrm{SNR}_{2}}{a_{2}}
\end{array}\right]
$$

Thus

$$
E[x \mid \mathbf{y}]=\gamma\left(\operatorname{SNR}_{1} \frac{y_{1}}{a_{1}}+\operatorname{SNR}_{2} \frac{y_{2}}{a_{2}}\right) .
$$

It follows that

$$
E[x \mid \mathbf{y}]=\boldsymbol{\mu}^{T} \mathbf{y},
$$

and in particular, $x$ can be written

$$
x=\boldsymbol{\mu}^{T} \mathbf{y}+\tilde{n},
$$

where $\tilde{n}$ is Gaussian, independent of $\mathbf{y}$, and has variance

$$
\begin{aligned}
& 1-\left[\begin{array}{ll}
a_{1} & a_{2}
\end{array}\right] \cdot \boldsymbol{K}_{y}^{-1} \cdot\left[\begin{array}{l}
a_{1} \\
a_{2}
\end{array}\right]=1-\left[\begin{array}{cc}
\frac{\gamma \mathrm{SNR}_{1}}{a_{1}} & \frac{\gamma \mathrm{SNR}_{2}}{a_{2}}
\end{array}\right]\left[\begin{array}{l}
a_{1} \\
a_{2}
\end{array}\right] \\
& =\gamma \text {. }
\end{aligned}
$$

Then for any random variable $u$ such that $x \leftrightarrow \mathbf{y} \leftrightarrow u$, by a calculation similar to (22), we have

$$
E\left[(x-E[x \mid u])^{2}\right]=\gamma+E\left[\left(\boldsymbol{\mu}^{T} \mathbf{y}-E\left[\boldsymbol{\mu}^{T} \mathbf{y} \mid u\right]\right)^{2}\right]
$$

As in the proof of Corollary 1, it follows that any code that achieves distortion $d$ for the $\boldsymbol{\mu}$-sum problem must achieve distortion $d+\gamma$ for the CEO problem (33) and vice versa. The characterization of the rate region in (29) and the sum rate in (30) now follow from existing results on the CEO problem [8, 10]. To show that (31) equals (30), we first show that (30) can be rewritten as

$$
\begin{gathered}
\inf \left\{\frac{1}{2} \log \left[1+\sum_{j=1}^{2} \operatorname{SNR}_{j}\left(1-2^{-2 r_{j}}\right)\right]+r_{1}+r_{2}: r_{1} \geq 0, r_{2} \geq 0,\right. \text { and } \\
\left.1+\sum_{j=1}^{2} \operatorname{SNR}_{j}\left(1-2^{-2 r_{j}}\right) \geq \frac{1}{d+\gamma}\right\}
\end{gathered}
$$

To see this, note that the two optimization problems differ only in the objective, and both objectives are increasing functions of $r_{1}$ and $r_{2}$. Now if $d>1-\gamma$, then 
both infima are zero. On the other hand, if $d \leq 1-\gamma$, then in both problems, we may assume without loss of generality that the constraint is met with equality

$$
1+\sum_{j=1}^{2} \operatorname{SNR}_{j}\left(1-2^{-2 r_{j}}\right)=\frac{1}{d+\gamma} .
$$

But if the constraint is met with equality, then the two objectives are equal. Thus the two optimization problems are equivalent.

Let $\mathbf{u}$ be a distributed Gaussian test channel such that

$$
x \leftrightarrow \mathbf{y} \leftrightarrow \mathbf{u} .
$$

If we define

$$
r_{j}=I\left(y_{j} ; u_{j} \mid x\right) \quad j \in\{1,2\},
$$

then a standard calculation shows that

$$
E\left[(x-E[x \mid \mathbf{u}])^{2}\right]=\left[1+\sum_{j=1}^{2} \operatorname{SNR}_{j}\left(1-2^{-2 r_{j}}\right)\right]^{-1} .
$$

Thus the expression in (35) equals

$$
\begin{aligned}
\inf \left\{I(x ; \mathbf{u})+I\left(y_{1} ; u_{1} \mid x\right)+I\left(y_{2} ; u_{2} \mid x\right)\right. & :(x, \mathbf{y}, \mathbf{u}) \text { are jointly Gaussian, } \\
& x \leftrightarrow \mathbf{y} \leftrightarrow \mathbf{u} \\
& u_{1} \leftrightarrow y_{1} \leftrightarrow y_{2} \leftrightarrow u_{2} \\
& \left.E\left[(x-E[x \mid \mathbf{u}])^{2}\right] \leq d+\gamma\right\} .
\end{aligned}
$$

Now since $\left(y_{1}, u_{1}\right) \leftrightarrow x \leftrightarrow\left(y_{2}, u_{2}\right)$ and $x \leftrightarrow \mathbf{y} \leftrightarrow \mathbf{u}$, we have

$$
\begin{aligned}
I(x ; \mathbf{u})+I\left(y_{1} ; u_{1} \mid x\right)+I\left(y_{2} ; u_{2} \mid x\right) & =I(x ; \mathbf{u})+I(\mathbf{y} ; \mathbf{u} \mid x) \\
& =I(x, \mathbf{y} ; \mathbf{u}) \\
& =I(\mathbf{y} ; \mathbf{u}) .
\end{aligned}
$$

Applying (34) again, we can write the infimum in (36) as

$$
\begin{aligned}
\inf \{I(\mathbf{y} ; \mathbf{u}): & (\mathbf{y}, \mathbf{u}) \text { are jointly Gaussian, } \\
& u_{1} \leftrightarrow y_{1} \leftrightarrow y_{2} \leftrightarrow u_{2} \\
& \left.E\left[\left(\boldsymbol{\mu}^{T} \mathbf{y}-E\left[\boldsymbol{\mu}^{T} \mathbf{y} \mid \mathbf{u}\right]\right)^{2}\right] \leq d\right\},
\end{aligned}
$$

which equals

$$
\inf \left\{\frac{1}{2} \log \frac{\left|\boldsymbol{K}_{y}\right|}{|\boldsymbol{D}|}: \boldsymbol{D} \in \mathcal{D}_{G} \text { and } \boldsymbol{\mu}^{T} \boldsymbol{D} \boldsymbol{\mu} \leq d\right\}
$$


Now since

$$
1+\sum_{j=1}^{2} \operatorname{SNR}_{j}\left(1-2^{-2 r_{j}}\right)
$$

is strictly concave, it follows that the infimum in (30) is achieved by a unique feasible point. This in turn implies that the infimum in (35) is achieved by a unique feasible point. By the equivalence between the feasible points in (35) and (38) it follows that the infimum in (38) is also achieved by a unique feasible point.

\section{Every $D^{*}$ Solves a $\mu$-sum Problem}

Proof of Lemma 4. Without loss of generality, we may assume that $\boldsymbol{\mu}^{*}$ has been scaled so that it satisfies the normalization (25). Then the sum rate for the $\boldsymbol{\mu}^{*}$ sum problem with allowable distortion

$$
d^{*}:=\boldsymbol{\mu}^{* T} \boldsymbol{D}^{*} \boldsymbol{\mu}^{*}
$$

is given by

$$
\begin{gathered}
\inf \left\{\frac{1}{2} \log \left[\frac{1}{d^{*}+\gamma}\right]+r_{1}+r_{2}: r_{1} \geq 0, r_{2} \geq 0,\right. \text { and } \\
\left.1+\sum_{j=1}^{2} \operatorname{SNR}_{j}\left(1-2^{-2 r_{j}}\right) \geq \frac{1}{d^{*}+\gamma}\right\},
\end{gathered}
$$

where $\mathrm{SNR}_{1}, \mathrm{SNR}_{2}$, and $\gamma$ were defined in equations (26) through (28). The remainder of the proof consists of three parts:

(I) We identify candidate optimizers for (39), $r_{1}^{*}$ and $r_{2}^{*}$, in terms of $\boldsymbol{D}^{*}$.

(II) We show that $r_{1}^{*}$ and $r_{2}^{*}$ achieve the infimum in (39).

(III) We show that at $r_{1}^{*}$ and $r_{2}^{*}$, the objective

$$
\frac{1}{2} \log \left[\frac{1}{d^{*}+\gamma}\right]+r_{1}^{*}+r_{2}^{*}
$$

equals

$$
\frac{1}{2} \log \frac{\left|\boldsymbol{K}_{y}\right|}{\left|\boldsymbol{D}^{*}\right|}
$$

Part I. Since $\boldsymbol{D}^{*}$ is in $\mathcal{D}_{G}$, there exists $\lambda_{1}^{*} \geq 0$ and $\lambda_{2}^{*} \geq 0$, such that

$$
\boldsymbol{D}^{*-1}=\boldsymbol{K}_{y}^{-1}+\left[\begin{array}{cc}
\lambda_{1}^{*} & 0 \\
0 & \lambda_{2}^{*}
\end{array}\right] \text {. }
$$


Our candidate optimizers are then

$$
r_{j}^{*}=\frac{1}{2} \log \left(1+\frac{\lambda_{j}^{*}}{1+\mathrm{SNR}_{j}}\right) \quad j \in\{1,2\} .
$$

This formula can be understood as follows. Since $\boldsymbol{D}^{*}$ is in $\mathcal{D}_{G}$, there exists a distributed Gaussian test channel $\mathbf{u}^{*}$ such that $\operatorname{Cov}\left(\mathbf{y} \mid \mathbf{u}^{*}\right)=\boldsymbol{D}^{*}$. Now $\mathbf{u}^{*}$ can be written

$$
u_{j}=\sqrt{\frac{\lambda_{j}^{*}}{1+\lambda_{j}^{*}}} \cdot y_{j}+z_{j} \quad j \in\{1,2\}
$$

where $\mathbf{z}$ is an independent Gaussian vector with covariance matrix

$$
\left[\begin{array}{cc}
\left(1+\lambda_{1}^{*}\right)^{-1} & 0 \\
0 & \left(1+\lambda_{2}^{*}\right)^{-1}
\end{array}\right] .
$$

As in the previous appendix, let

$$
a_{j}=\left(\frac{\mathrm{SNR}_{j}}{1+\mathrm{SNR}_{j}}\right)^{1 / 2} \quad j \in\{1,2\}
$$

and let $x, n_{1}$, and $n_{2}$ be zero mean Gaussian random variables with

$$
\begin{aligned}
& E\left[x^{2}\right]=1 \\
& E\left[n_{j}^{2}\right]=1-a_{j}^{2} \quad j \in\{1,2\} .
\end{aligned}
$$

Then couple these variables to $\left(\mathbf{y}, \mathbf{u}^{*}\right)$ such that

$$
\begin{aligned}
& y_{1}=a_{1} x+n_{1} \\
& y_{2}=a_{2} x+n_{2}
\end{aligned}
$$

and $x \leftrightarrow \mathbf{y} \leftrightarrow \mathbf{u}^{*}$. It then follows that

$$
\begin{aligned}
r_{j}^{*} & =\frac{1}{2} \log \left(1+\lambda_{j}^{*}\left(1-a_{j}^{2}\right)\right) \\
& =I\left(y_{j} ; u_{j}^{*} \mid x\right) .
\end{aligned}
$$

Part II. Next we show that $r_{1}^{*}$ and $r_{2}^{*}$ solve the optimization problem (39). Since the optimization problem is convex, it suffices to show that $r_{1}^{*}$ and $r_{2}^{*}$ satisfy the Karush-Kuhn-Tucker (KKT) conditions [21, Section 5.5.3]. The Lagrangian for this optimization problem is

$$
\begin{aligned}
& L\left(r_{1}, r_{2}, \nu\right) \\
& \quad=\frac{1}{2} \log \left[\frac{1}{d^{*}+\gamma}\right]+r_{1}+r_{2}-\nu\left(1+\sum_{j=1}^{2} \operatorname{SNR}_{j}\left(1-2^{-2 r_{j}}\right)-\frac{1}{d^{*}+\gamma}\right) .
\end{aligned}
$$


Thus it suffices to show that

$$
\begin{aligned}
2^{2 r_{j}^{*}} & =\nu^{*} \operatorname{SNR}_{j} \quad \forall j \in\{1,2\} \\
1+\sum_{j=1}^{2} \operatorname{SNR}_{j}\left(1-2^{-2 r_{j}^{*}}\right) & \geq \frac{1}{d^{*}+\gamma}
\end{aligned}
$$

for some $\nu^{*} \geq 0$. To show (42), note that from (40),

$$
\boldsymbol{D}^{*}=\left(1-\rho^{2}\right)\left[\begin{array}{cc}
1+\left(1-\rho^{2}\right) \lambda_{1}^{*} & -\rho \\
-\rho & 1+\left(1-\rho^{2}\right) \lambda_{2}^{*}
\end{array}\right]^{-1} .
$$

Then define

$$
s_{j}^{*}=1+\left(1-\rho^{2}\right) \lambda_{j}^{*} \quad j \in\{1,2\} .
$$

Since

$$
\boldsymbol{D}^{*}=\left[\begin{array}{cc}
d_{1} & \theta^{*} \sqrt{d_{1} d_{2}} \\
\theta^{*} \sqrt{d_{1} d_{2}} & d_{2}
\end{array}\right],
$$

it follows from (44) that

$$
\frac{d_{1}}{d_{2}}=\frac{s_{2}^{*}}{s_{1}^{*}}
$$

Thus

$$
\begin{aligned}
& \mathrm{SNR}_{1}=\frac{\rho}{1-\rho^{2}}\left(\sqrt{\frac{s_{1}^{*}}{s_{2}^{*}}}+\rho\right) \\
& \mathrm{SNR}_{2}=\frac{\rho}{1-\rho^{2}}\left(\sqrt{\frac{s_{2}^{*}}{s_{1}^{*}}}+\rho\right) .
\end{aligned}
$$

Combining (41) and (45), we have

$$
s_{j}^{*}=\left(1-\rho^{2}\right)\left(1+\mathrm{SNR}_{j}\right)\left(2^{2 r_{j}^{*}}-1\right)+1 .
$$

Thus (42) is equivalent to

$$
\frac{s_{j}^{*}-1}{\left(1-\rho^{2}\right)\left(1+\mathrm{SNR}_{j}\right)}+1=\nu^{*} \operatorname{SNR}_{j} \quad \forall j \in\{1,2\}
$$

But by using (46) and (47), one can verify that this pair of conditions holds if

$$
\nu^{*}=\frac{1-\rho^{2}}{\rho} \frac{s_{1}^{*} s_{2}^{*}+\rho \sqrt{s_{1}^{*} s_{2}^{*}}}{\rho\left(s_{1}^{*}+s_{2}^{*}\right)+\sqrt{s_{1}^{*} s_{2}^{*}}\left(1+\rho^{2}\right)} .
$$

This establishes (48) and hence (42). Now as in the previous appendix we have

$$
\begin{aligned}
E\left[\left(x-E\left[x \mid \mathbf{u}^{*}\right]\right)^{2}\right] & =\left[1+\sum_{j=1}^{2} \operatorname{SNR}_{j}\left(1-2^{-2 r_{j}^{*}}\right)\right]^{-1} \\
& =\gamma+E\left[\left(\boldsymbol{\mu}^{* T} \mathbf{y}-E\left[\boldsymbol{\mu}^{* T} \mathbf{y} \mid \mathbf{u}^{*}\right]\right)^{2}\right] \\
& =\gamma+\boldsymbol{\mu}^{* T} \boldsymbol{D}^{*} \boldsymbol{\mu}^{*} \\
& =\gamma+d^{*} .
\end{aligned}
$$


This establishes (43) and the optimality of $r_{1}^{*}$ and $r_{2}^{*}$.

Part III. It only remains to show that

$$
\frac{1}{2} \log \left[\frac{1}{d^{*}+\gamma}\right]+r_{1}^{*}+r_{2}^{*}=\frac{1}{2} \log \frac{\left|\boldsymbol{K}_{y}\right|}{\left|\boldsymbol{D}^{*}\right|} .
$$

Observe that the left-hand side equals

$$
I\left(x ; \mathbf{u}^{*}\right)+\sum_{j=1}^{2} I\left(y_{j} ; u_{j}^{*} \mid x\right) .
$$

Repeating the argument in (37), we have

$$
I\left(x ; \mathbf{u}^{*}\right)+\sum_{j=1}^{2} I\left(y_{j} ; u_{j}^{*} \mid x\right)=I\left(\mathbf{y} ; \mathbf{u}^{*}\right)=\frac{1}{2} \log \frac{\left|\boldsymbol{K}_{y}\right|}{\left|\boldsymbol{D}^{*}\right|} .
$$

\section{E Achievability for $M$-sums}

Before proving Lemma 7, we examine some of the properties of the constituent regions $\mathcal{R}_{j}^{\star}\left(d_{j}\right)$. Without loss of generality, we focus on $\mathcal{R}_{1}^{\star}\left(d_{1}\right)$.

Let $h_{1}(\cdot)$ denote the function whose epigraph is $\mathcal{R}_{1}^{\star}\left(d_{1}\right)$

$$
\mathcal{R}_{1}^{\star}\left(d_{1}\right)=\left\{\left(R_{1}, R_{2}\right): R_{2} \geq h_{1}\left(R_{1}\right)\right\},
$$

which may equal infinity for some $R_{1}$. Note that $h_{1}(\cdot)$ is nonincreasing. Since $\mathcal{R}_{1}^{\star}\left(d_{1}\right)$ is closed and convex, $h_{1}(\cdot)$ must be continuous on its effective domain [22, Theorems 7.1 and 10.1]. Thus $h_{1}(\cdot)$ is closed and proper [22].

For $R_{1}$ in the effective domain of $R_{1}$, let $\partial h_{1}\left(R_{1}\right)$ denote the subdifferential of $h_{1}(\cdot)$ at $R_{1}$

$$
\partial h_{1}\left(R_{1}\right)=\left\{s: h_{1}(R) \geq h_{1}\left(R_{1}\right)+s\left(R-R_{1}\right) \forall R\right\} .
$$

Note that $\partial h_{1}\left(R_{1}\right)$ can be interpreted geometrically as the set of slopes of all supporting lines at $R_{1}$. From standard results in convex analysis [22, Section 24], $\partial h_{1}\left(R_{1}\right)$ is the interval between the left and right derivatives of $h_{1}(\cdot)$ at $R_{1}$. The graph of $\partial h_{1}(\cdot)$

$$
\left\{(R, s): s \in \partial h_{1}(R)\right\}
$$

resembles the graph of a nondecreasing function, except that any jump discontinuities have been "filled in" with vertical segments. As such, $\partial h_{1}(\cdot)$ is monotonic in the sense that if $R_{1} \leq \tilde{R}_{1}$ then $s \leq \tilde{s}$ for any $s$ in $\partial h_{1}\left(R_{1}\right)$ and $\tilde{s}$ in $\partial h_{1}\left(\tilde{R}_{1}\right)$.

We now partition the effective domain of $h_{1}(\cdot)$ into three parts. Let

$$
\begin{aligned}
W & =\left\{R_{1}: \max \left(\partial h_{1}\left(R_{1}\right)\right)<-1\right\} \\
X & =\left\{R_{1}: \min \left(\partial h_{1}\left(R_{1}\right)\right) \leq-1 \leq \max \left(\partial h_{1}\left(R_{1}\right)\right)\right\} \\
Y & =\left\{R_{1}:-1<\min \left(\partial h_{1}\left(R_{1}\right)\right)<0\right\} \\
Z & =\left\{R_{1}: \partial h_{1}\left(R_{1}\right)=\{0\}\right\} .
\end{aligned}
$$


These intervals are depicted in Fig. 7. We call $W$ the steep part of $\mathcal{R}_{1}^{\star}\left(d_{1}\right)$ and $Y$ and $Z$ the shallow part. Some of these intervals might be empty in some cases. Note that the sum rate decreases as one moves left-to-right in $W$ and increases as one moves left-to-right in $Y$ and $Z$.

Next we associate each point $R_{1}$ in the effective domain of $h_{1}(\cdot)$ with a test channel that meets the $\boldsymbol{\mu}_{1}$ distortion constraint with equality. Since $\left(R_{1}, h_{1}\left(R_{1}\right)\right)$ is on the boundary of $\mathcal{R}_{1}^{\star}\left(d_{1}\right)$, it must be on the boundary of the contrapolymatroid of some test channel $\boldsymbol{D}$ satisfying $\boldsymbol{\mu}_{1}^{T} \boldsymbol{D} \boldsymbol{\mu}_{1}=d_{1}$. Suppose first that $R_{1}$ is in $W$. Then $\mathcal{R}_{1}^{\star}\left(d_{1}\right)$ has a supporting line with slope $s<-1$ at $\left(R_{1}, h_{1}\left(R_{1}\right)\right)$. Since the contrapolymatroid associated with $\boldsymbol{D}$ is contained in $\mathcal{R}_{1}^{\star}\left(d_{1}\right)$, this contrapolymatroid must also be supported by a line with slope $s<-1$ at $\left(R_{1}, h_{1}\left(R_{1}\right)\right)$. This implies that $\left(R_{1}, h_{1}\left(R_{1}\right)\right)$ is on the vertical portion of the boundary of this contrapolymatroid.

In fact, by the definition of $h_{1}(\cdot),\left(R_{1}, h_{1}\left(R_{1}\right)\right)$ must be the left corner point of the contrapolymatroid of this test channel. We then associate $R_{1}$ with the unique test channel whose left corner point is $\left(R_{1}, h_{1}\left(R_{1}\right)\right)$. Likewise, to every $R_{1}$ in $Y$, we associate the unique test channel whose right corner point is $\left(R_{1}, h_{1}\left(R_{1}\right)\right)$.

If $Z$ is nonempty then $X \cup Y$ is bounded. In this case, as $R_{1} \rightarrow \sup X \cup Y$, the associated test channels will converge to a test channel $\boldsymbol{D}$. We associate this test channel with all $R_{1}$ in $Z$.

If $R_{1}$ is in $X$, then $\mathcal{R}_{1}^{\star}\left(d_{1}\right)$ is supported at $\left(R_{1}, h_{1}\left(R_{1}\right)\right)$ by a line with slope -1 . This implies that $\left(R_{1}, h_{1}\left(R_{1}\right)\right)$ is sum-rate optimal. We then associate all $R_{1}$ in $X$ with the unique test channel that is sum-rate optimal (see Lemma 8).

Note that the end-points of the interval $X$ must correspond to the corner points of the sum-rate optimal contrapolymatroid. It follows that the associated test-channels vary continuously with $R_{1}$ over the entire effective domain of $h_{1}(\cdot)$. The test channels also vary monotonically in the sense that if $R_{1} \leq \tilde{R}_{1}$ and $\boldsymbol{\Lambda}$ and $\tilde{\boldsymbol{\Lambda}}$ are the associated test channels, then

$$
\boldsymbol{\Lambda}=\tilde{\boldsymbol{\Lambda}}+\left[\begin{array}{cc}
-\lambda_{1} & 0 \\
0 & \lambda_{2}
\end{array}\right]
$$

for some nonnegative numbers $\lambda_{1}$ and $\lambda_{2}$. Note that for each test channel that meets the distortion constraint with equality, at least one of its corner points must be on the boundary of $\mathcal{R}_{1}^{\star}\left(d_{1}\right)$.

Now consider a second vector, $\boldsymbol{\mu}_{2}$, and suppose that $\boldsymbol{\mu}_{2}$ weights $y_{2}$ more heavily than $\boldsymbol{\mu}_{1}$ does

$$
\frac{\mu_{22}}{\mu_{21}}>\frac{\mu_{12}}{\mu_{11}} .
$$

Next we show that as one moves left-to-right along the boundary of $\mathcal{R}_{1}^{\star}\left(d_{1}\right)$, the distortion that the associated test channels induce on $\boldsymbol{\mu}_{2}^{T} \mathbf{y}$ is nondecreasing.

Lemma 9. Suppose $R_{1} \leq \tilde{R}_{1}$, and let $\boldsymbol{D}$ and $\tilde{\boldsymbol{D}}$ be the associated test channels. Then

$$
\boldsymbol{\mu}_{2}^{T} \boldsymbol{D} \boldsymbol{\mu}_{2} \leq \boldsymbol{\mu}_{2}^{T} \tilde{\boldsymbol{D}} \boldsymbol{\mu}_{2} .
$$


Proof. Define $\boldsymbol{\Lambda}$ and $\tilde{\boldsymbol{\Lambda}}$ by

$$
\begin{aligned}
& \boldsymbol{D}^{-1}=\boldsymbol{K}_{y}^{-1}+\boldsymbol{\Lambda} \\
& \tilde{\boldsymbol{D}}^{-1}=\boldsymbol{K}_{y}^{-1}+\tilde{\boldsymbol{\Lambda}}
\end{aligned}
$$

Since $R_{1} \leq \tilde{R}_{1}$, we know that

$$
\boldsymbol{\Lambda}=\tilde{\boldsymbol{\Lambda}}+\left[\begin{array}{cc}
-\lambda_{1} & 0 \\
0 & \lambda_{2}
\end{array}\right]
$$

for some nonnegative numbers $\lambda_{1}$ and $\lambda_{2}$. Then

$$
\boldsymbol{D}^{-1}=\tilde{\boldsymbol{D}}^{-1}+\left[\begin{array}{cc}
-\lambda_{1} & 0 \\
0 & \lambda_{2}
\end{array}\right]
$$

Furthermore, we know that

$$
\boldsymbol{\mu}_{1}^{T} \boldsymbol{D} \boldsymbol{\mu}_{1}=\boldsymbol{\mu}_{1}^{T} \tilde{D} \boldsymbol{\mu}_{1}=d_{1} .
$$

To establish (50), we will show that

$$
0 \leq \boldsymbol{\mu}_{2}^{T} \tilde{\boldsymbol{D}} \boldsymbol{\mu}_{2} \boldsymbol{\mu}_{1}^{T} \boldsymbol{D} \boldsymbol{\mu}_{1}-\boldsymbol{\mu}_{1}^{T} \tilde{\boldsymbol{D}} \boldsymbol{\mu}_{1} \boldsymbol{\mu}_{2}^{T} \boldsymbol{D} \boldsymbol{\mu}_{2}
$$

Write

$$
\tilde{\boldsymbol{D}}^{-1}=\left[\begin{array}{cc}
a & -b \\
-b & c
\end{array}\right]
$$

where $a, b$, and $c$ are nonnegative. Then

$$
\begin{aligned}
\tilde{\boldsymbol{D}} & =\frac{1}{a c-b^{2}}\left[\begin{array}{ll}
c & b \\
b & a
\end{array}\right] \\
\boldsymbol{D} & =\frac{1}{\left(a-\lambda_{1}\right)\left(c+\lambda_{2}\right)-b^{2}}\left[\begin{array}{cc}
c+\lambda_{2} & b \\
b & a-\lambda_{1}
\end{array}\right]
\end{aligned}
$$

A tedious but straightforward calculation shows that the expression on the righthand side of (51) equals

$$
\frac{\left(\lambda_{1} c+a \lambda_{2}\right)\left(\mu_{11}^{2} \mu_{22}^{2}-\mu_{12}^{2} \mu_{21}^{2}\right)+2 b\left(\lambda_{1} \mu_{12} \mu_{22}+\lambda_{2} \mu_{11} \mu_{21}\right)\left(\mu_{11} \mu_{22}-\mu_{12} \mu_{21}\right)}{\left(a c-b^{2}\right)\left(\left(a-\lambda_{1}\right)\left(c+\lambda_{2}\right)-b^{2}\right)}
$$

which is nonnegative because $|\boldsymbol{D}|>0,|\tilde{\boldsymbol{D}}|>0$, and

$$
\mu_{11} \mu_{22}-\mu_{12} \mu_{21}>0
$$

by (49).

From the proof, we can see that if the inequality in (49) is reversed, then the inequality in (50) is also reversed. 
We now turn to the proof that

$$
\mathcal{R}_{\text {sum }}^{\star}\left(d_{1}, \ldots, d_{J}\right) \cap \bigcap_{j=1}^{J} \mathcal{R}_{j}^{\star}\left(d_{j}\right)
$$

is achievable. Recall that $\mathcal{R}_{\text {sum }}^{\star}\left(d_{1}, \ldots d_{J}\right)$ is defined as

$$
\mathcal{R}_{\text {sum }}^{\star}\left(d_{1}, \ldots, d_{J}\right)=\left\{\left(R_{1}, R_{2}\right): R_{1}+R_{2} \geq R_{\text {sum }}^{\star}\left(d_{1}, \ldots, d_{J}\right)\right\}
$$

where

$$
\begin{aligned}
& R_{\text {sum }}^{\star}\left(d_{1}, \ldots, d_{J}\right) \\
& \quad=\inf \left\{\frac{1}{2} \log \frac{\left|\boldsymbol{K}_{y}\right|}{|\boldsymbol{D}|}: \boldsymbol{D} \in \mathcal{D}_{G} \text { and } \boldsymbol{\mu}_{j}^{T} \boldsymbol{D} \boldsymbol{\mu}_{j} \leq d_{j} \forall j \in\{1, \ldots, J\}\right\} .
\end{aligned}
$$

We begin by showing that this infimum is achieved.

Lemma 10. There exists a $\boldsymbol{D}^{*}$ in $\mathcal{D}_{G}$ such that

$$
\boldsymbol{\mu}_{j}^{T} \boldsymbol{D}^{*} \boldsymbol{\mu}_{j} \leq d_{j} \quad \forall j \in\{1, \ldots, J\}
$$

and

$$
\frac{1}{2} \log \frac{\left|\boldsymbol{K}_{y}\right|}{\left|\boldsymbol{D}^{*}\right|}=\inf \left\{\frac{1}{2} \log \frac{\left|\boldsymbol{K}_{y}\right|}{|\boldsymbol{D}|}: \boldsymbol{D} \in \mathcal{D}_{G} \text { and } \boldsymbol{\mu}_{j}^{T} \boldsymbol{D} \boldsymbol{\mu}_{j} \leq d_{j} \forall j \in\{1, \ldots, J\}\right\} .
$$

Proof. Let $\tilde{\boldsymbol{D}}$ by any matrix in $\mathcal{D}_{G}$ that satisfies the distortion constraints. The infimum is then upper bounded by

$$
\frac{1}{2} \log \frac{\left|\boldsymbol{K}_{y}\right|}{|\tilde{\boldsymbol{D}}|} .
$$

Now the set of $\boldsymbol{D} \in \mathcal{D}_{G}$ such that

$$
\begin{aligned}
\boldsymbol{\mu}_{j}^{T} \boldsymbol{D} \boldsymbol{\mu}_{j} & \leq d_{j} \quad \forall j \in\{1, \ldots, J\} \\
\frac{1}{2} \log \frac{\left|\boldsymbol{K}_{y}\right|}{|\boldsymbol{D}|} & \leq \frac{1}{2} \log \frac{\left|\boldsymbol{K}_{y}\right|}{|\tilde{\boldsymbol{D}}|},
\end{aligned}
$$

is compact, and the objective is continuous, so the infimum is achieved.

Proof of Lemma 7. Recall we are assuming that the vectors $\boldsymbol{\mu}_{1}, \ldots, \boldsymbol{\mu}_{J}$ are distinct and have norm one. We may also assume without loss of generality that they have been ordered so that their first coordinates are decreasing

$$
\mu_{11}>\mu_{21}>\cdots>\mu_{J 1}
$$

which implies that their second coordinates are increasing

$$
\mu_{12}<\mu_{22}<\cdots<\mu_{J 2} .
$$


Let

$$
h_{j}\left(R_{1}\right)=\inf \left\{\left(R_{2}:\left(R_{1}, R_{2}\right) \in \mathcal{R}_{j}^{\star}\left(d_{j}\right)\right\} \quad j \in\{1, \ldots, J\}\right.
$$

and

$$
h_{\text {sum }}\left(R_{1}\right)=\inf \left\{\left(R_{2}:\left(R_{1}, R_{2}\right) \in \mathcal{R}_{\text {sum }}^{\star}\left(d_{1}, d_{2}, \ldots, d_{J}\right)\right\}\right.
$$

denote the functions whose epigraphs are the constituent regions. The corresponding function for the intersection is given by

$$
h\left(R_{1}\right)=\max \left(h_{1}\left(R_{1}\right), \ldots, h_{J}\left(R_{1}\right), h_{\mathrm{sum}}\left(R_{1}\right)\right) .
$$

To show that the intersection is achievable, is suffices to show that for each $R_{1}$ for which $h\left(R_{1}\right)<\infty,\left(R_{1}, h_{j}\left(R_{1}\right)\right)$ is achievable for some $j$ or $\left(R_{1}, h_{\text {sum }}\left(R_{1}\right)\right)$ is achievable.

By Lemma 10 , there exists a $\boldsymbol{D}^{*}$ in $\mathcal{D}_{G}$ that is sum-rate optimal within this class

$$
\begin{aligned}
\boldsymbol{\mu}_{j}^{T} \boldsymbol{D}^{*} \boldsymbol{\mu}_{j} & \leq d_{j} \forall j \in\{1, \ldots, J\} \\
\frac{1}{2} \log \frac{\left|\boldsymbol{K}_{y}\right|}{\left|\boldsymbol{D}^{*}\right|} & =\inf \left\{\frac{1}{2} \log \frac{\left|\boldsymbol{K}_{y}\right|}{|\boldsymbol{D}|}: \boldsymbol{D} \in \mathcal{D}_{G} \text { and } \boldsymbol{\mu}_{j}^{T} \boldsymbol{D} \boldsymbol{\mu}_{j} \leq d_{j} \forall j \in\{1, \ldots, J\}\right\} .
\end{aligned}
$$

Let $A$ denote the set of constraints that are active at $\boldsymbol{D}^{*}$

$$
A=\left\{j: \boldsymbol{\mu}_{j}^{T} \boldsymbol{D}^{*} \boldsymbol{\mu}_{j}=d_{j}\right\},
$$

which must be nonempty. Let $i$ denote the largest element of $A$. Thus the $\boldsymbol{\mu}_{i}$ sum is the one that weights $y_{2}$ most heavily of all of the sums whose constraints are active at $\boldsymbol{D}^{*}$.

Consider the contrapolymatroid region associated with $\boldsymbol{D}^{*}$. We shall show that the right corner point of this region is on the boundary of $\mathcal{R}_{i}^{\star}\left(d_{i}\right)$. Since $\boldsymbol{D}^{*}$ achieves the $\boldsymbol{\mu}_{i}$ constraint with equality, either the left corner point or the right corner point (or both) of its contrapolymatroid region must lie on the boundary of $\mathcal{R}_{i}^{\star}\left(d_{i}\right)$. Suppose it is only the left corner point that lies on the boundary of $\mathcal{R}_{i}^{\star}\left(d_{i}\right)$. This corner point would then have to lie on the steep part of the boundary $(W)$ of $\mathcal{R}_{i}^{\star}\left(d_{i}\right)$. Consider moving a small amount to the right along the boundary of $\mathcal{R}_{i}^{\star}\left(d_{i}\right)$. Since the distortion for $\boldsymbol{\mu}_{j}$ varies continuously as we move along the boundary of $\mathcal{R}_{i}^{\star}\left(d_{i}\right)$ for each $j$, for a sufficiently small movement, none of the distortion constraints in $A^{c}$ will be violated. At the same time, by Lemma 9, none of the distortion constraints in $A$ will be violated either.

Thus we can strictly reduce the sum rate without violating any of the distortion constraints. This contradicts the assumption that $\boldsymbol{D}^{*}$ is sum-rate optimal within the class $\mathcal{D}_{G}$. Hence it must be the right corner point, $\left(R_{1}^{c}, R_{2}^{c}\right)$, of the contrapolymatroid that is on the boundary of $\mathcal{R}_{i}^{\star}\left(d_{i}\right)$, as shown in Fig. 8.

Next consider starting at this corner point and moving to the right along the boundary of $\mathcal{R}_{i}^{\star}\left(d_{i}\right)$. By Lemma 9 , the $\boldsymbol{\mu}_{j}$ constraint will remain satisfied for all $j \leq i$. If, as we move to the right, the $\boldsymbol{\mu}_{k}$ constraint is never satisfied with equality for all $k>i$, then it follows that the shallow portion of $\mathcal{R}_{i}^{\star}\left(d_{i}\right)$ to the right of $\left(R_{1}^{c}, R_{2}^{c}\right)$ is achievable. 
If the $\boldsymbol{\mu}_{k}$ distortion constraint becomes active for some $k>i$, then there exists a point $\left(\tilde{R}_{1}, \tilde{R}_{2}\right)$ on the boundary of $\mathcal{R}_{i}^{\star}\left(d_{i}\right)$ whose associated test channel $\tilde{\boldsymbol{D}}$ meets both the $\boldsymbol{\mu}_{i}$ and $\boldsymbol{\mu}_{k}$ distortion constraints with equality

$$
\begin{aligned}
\boldsymbol{\mu}_{i}^{T} \tilde{\boldsymbol{D}} \boldsymbol{\mu}_{i} & =d_{i} \\
\boldsymbol{\mu}_{k}^{T} \tilde{\boldsymbol{D}} \boldsymbol{\mu}_{k} & =d_{k} .
\end{aligned}
$$

Since $\left(\tilde{R}_{1}, \tilde{R}_{2}\right)$ is on the shallow portion of the boundary of $\mathcal{R}_{i}^{\star}\left(d_{i}\right),\left(\tilde{R}_{1}, \tilde{R}_{2}\right)$ must be the right corner point of the contrapolymatroid region associated with $\tilde{\boldsymbol{D}}$. Now at least one of the corner points of $\tilde{\boldsymbol{D}}$ must be on the boundary of $\mathcal{R}_{k}^{\star}\left(d_{k}\right)$. By Lemma 9, the boundary of $\mathcal{R}_{k}^{\star}\left(d_{k}\right)$ must be below that of $\mathcal{R}_{i}^{\star}\left(d_{i}\right)$ to the left of $\tilde{R}_{1}$. It follows that it must be the right corner point of $\tilde{\boldsymbol{D}}$, i.e., $\left(\tilde{R}_{1}, \tilde{R}_{2}\right)$, that is on the boundary of $\mathcal{R}_{k}^{\star}\left(d_{k}\right)$. We then move to the right along the boundary of $\mathcal{R}_{k}^{\star}\left(d_{k}\right)$, repeating the above process as necessary as new constraints become active. This shows that for each $R_{1} \geq R_{1}^{c},\left(R_{1}, h_{j}\left(R_{1}\right)\right)$ is achievable for some $j$.

An analogous procedure can be followed starting with the left corner point of the contrapolymatroid associated with $\boldsymbol{D}^{*}$. Finally, between these two corner points, $\left(R_{1}, h_{\text {sum }}\left(R_{1}\right)\right)$ is achievable.

\section{F Converse for $M$-sums}

We show in this appendix that the rate region for the $\boldsymbol{M}$-sums problem is contained in

$$
\mathcal{R}_{\text {sum }}^{\star}\left(d_{1}, \ldots, d_{J}\right) \cap \bigcap_{j=1}^{J} \mathcal{R}_{j}^{\star}\left(d_{j}\right) .
$$

Since the rate region is clearly contained in $\cap_{j=1}^{J} \mathcal{R}_{j}^{\star}\left(d_{j}\right)$, we only need to show that it is contained in $\mathcal{R}_{\text {sum }}^{\star}\left(d_{1}, \ldots, d_{J}\right)$. That is, we must show that the sum rate is lower bounded by

$$
\begin{aligned}
& R_{\text {sum }}^{\star}\left(d_{1}, \ldots, d_{J}\right) \\
& \quad=\inf \left\{\frac{1}{2} \log \frac{\left|\boldsymbol{K}_{y}\right|}{|\boldsymbol{D}|}: \boldsymbol{D} \in \mathcal{D}_{G} \text { and } \boldsymbol{\mu}_{j}^{T} \boldsymbol{D} \boldsymbol{\mu}_{j} \leq d_{j} \forall j \in\{1, \ldots, J\}\right\} .
\end{aligned}
$$

By Lemma 10, this infimum is achieved by some $\boldsymbol{D}^{*}$ in $\mathcal{D}_{G}$. If $\boldsymbol{D}^{*}=\boldsymbol{K}_{y}$, then $R_{\text {sum }}^{\star}\left(d_{1}, \ldots, d_{J}\right)=0$ and our task is trivial. Suppose therefore that $\boldsymbol{D}^{*} \neq \boldsymbol{K}_{y}$. Then $\boldsymbol{D}^{*}$ must meet at least one of the distortion constraints with equality. If $\boldsymbol{D}^{*}$ meets exactly one distortion constraint with equality, then the converse is relatively simple, because in this case $\mathcal{R}_{\text {sum }}^{\star}\left(d_{1}, \ldots, d_{J}\right)$ contains $\mathcal{R}_{j}^{\star}\left(d_{j}\right)$ for some $j$.

Lemma 11. Suppose there exists $\boldsymbol{D}^{*}$ in $\mathcal{D}_{G}$ that is sum-rate optimal within this class and meets exactly one of the distortion constraints with equality, i.e., 
$\boldsymbol{\mu}_{i}^{T} \boldsymbol{D}^{*} \boldsymbol{\mu}_{i}=d_{i}$ and $\boldsymbol{\mu}_{j}^{T} \boldsymbol{D}^{*} \boldsymbol{\mu}_{j}<d_{j}$ for all $j \neq i$. Then the sum rate for the $\boldsymbol{M}$-sums problem is

$$
\frac{1}{2} \log \frac{\left|\boldsymbol{K}_{y}\right|}{\left|\boldsymbol{D}^{*}\right|}=R_{\mathrm{sum}}^{\star}\left(d_{1}, \ldots, d_{J}\right) .
$$

Proof. It suffices to show that $\boldsymbol{D}^{*}$ is sum-rate optimal for the $\boldsymbol{\mu}_{i}$-sum problem with distortion $d_{i}$. Recall that one of the corner points of $\boldsymbol{D}^{*}$ lies on the boundary of the $\boldsymbol{\mu}_{i}$-sum rate region. If this point is not sum-rate optimal, then it is possible to move a small distance along the boundary of $\mathcal{R}_{i}^{\star}\left(d_{i}\right)$ and strictly decrease the sum rate. Since the distortion associated with $\boldsymbol{\mu}_{j}$ varies continuously as we move along this boundary for each $j$, it follows that a sufficiently small movement will not violate any of the distortion constraints. This contradicts the assumption that $\boldsymbol{D}^{*}$ is sum-rate optimal for the $\boldsymbol{M}$-sums problem within the class $\mathcal{D}_{G}$. It follows that $\boldsymbol{D}^{*}$ is sum-rate optimal for the $\boldsymbol{\mu}_{i}$-sum problem.

It also happens that $\mathcal{R}_{\text {sum }}^{\star}\left(d_{1}, \ldots, d_{J}\right)$ contains $\mathcal{R}_{j}^{\star}\left(d_{j}\right)$ for some $j$ when there is an optimizing $\boldsymbol{D}^{*}$ on the "boundary" of $\mathcal{D}_{G}$. Let $\mathcal{D}_{G}^{\circ}$ denote the set of $\boldsymbol{D}$ in $\mathcal{D}_{G}$ such that

$$
\boldsymbol{D}^{-1}=\boldsymbol{K}_{y}^{-1}+\boldsymbol{\Lambda}
$$

for some diagonal and positive definite $\Lambda$. Let $\partial \mathcal{D}=\mathcal{D}_{G}-\mathcal{D}_{G}^{\circ}$, which is the set of those $\boldsymbol{D} \in \mathcal{D}_{G}$ such that $\boldsymbol{D}^{-1}-\boldsymbol{K}_{y}^{-1}$ is singular.

Lemma 12. Suppose there exists a $\boldsymbol{D}^{*} \neq \boldsymbol{K}_{y}$ in $\partial \mathcal{D}_{G}$ that is sum-rate optimal within the class $\mathcal{D}_{G}$. Then the sum rate for the $\boldsymbol{M}$-sums problem is given by

$$
\frac{1}{2} \log \frac{\left|\boldsymbol{K}_{y}\right|}{\left|\boldsymbol{D}^{*}\right|}=R_{\text {sum }}^{\star}\left(d_{1}, \ldots, d_{J}\right) .
$$

Proof. As in the previous proof, it suffices to show that for some $i, \boldsymbol{D}^{*}$ is sumrate optimal for the $\boldsymbol{\mu}_{i}$-sum problem with distortion $d_{i}$. Now $\boldsymbol{D}^{*}$ can be written

$$
\boldsymbol{D}^{*}=\left(\boldsymbol{K}_{y}^{-1}+\boldsymbol{\Lambda}\right)^{-1}
$$

where $\boldsymbol{\Lambda}$ is diagonal, positive semidefinite, singular, and nonzero. Without loss of generality, we may assume that $\boldsymbol{\Lambda}$ is of the form

$$
\boldsymbol{\Lambda}=\left[\begin{array}{cc}
\lambda_{1} & 0 \\
0 & 0
\end{array}\right]
$$

for some $\lambda_{1}>0$. Let $A$ denote the set of constraints that are active at $\boldsymbol{D}^{*}$

$$
A=\left\{j: \boldsymbol{\mu}_{j}^{T} \boldsymbol{D}^{*} \boldsymbol{\mu}_{j}=d_{j}\right\}
$$

Let $i$ denote the constraint in $A$ that weights $y_{1}$ most heavily

$$
i=\arg \max _{j \in A} \frac{\mu_{j 1}}{\mu_{j 2}} .
$$


We will show that $\boldsymbol{D}^{*}$ is sum-rate optimal for the $\boldsymbol{\mu}_{i}$-sum problem with distortion $d_{i}$. Now $\boldsymbol{D}^{*}$ is associated with a point $\left(R_{1}, 0\right)$ on the boundary of the $\boldsymbol{\mu}_{i}$-sum rate region. If this point is not sum-rate optimal for this problem, then it is possible to move a small distance to the left along the boundary of $\mathcal{R}_{i}^{\star}\left(d_{i}\right)$ and strictly decrease the sum rate. Since the distortion associated with $\boldsymbol{\mu}_{j}$ varies continuously as one moves along this boundary, for a sufficiently small movement, none of the constraints in $A^{c}$ will be violated. On the other hand, by Lemma 9, none of the constraints in $A$ will be violated either. This contradicts the assumption that $\boldsymbol{D}^{*}$ is sum-rate optimal for the $\boldsymbol{M}$-sums problem within the class $\mathcal{D}_{G}$. It follows that $\boldsymbol{D}^{*}$ is sum-rate optimal for the $\boldsymbol{\mu}_{i}$-sum problem.

The previous two lemmas allows us to focus on the case in which there exists an optimizing $\boldsymbol{D}^{*}$ in $\mathcal{D}_{G}^{\circ}$ that meets at least two of the distortion constraints with equality. Our proof in this case parallels the proof of Theorem 1. In particular, we introduce a nonnegative vector $\boldsymbol{\mu}^{*}$ such that $\boldsymbol{D}^{*}$ is sum-rate optimal for the $\boldsymbol{\mu}^{*}$-sum problem.

Lemma 13. Suppose $\boldsymbol{D}^{*} \in \mathcal{D}_{G}^{\circ}$ is sum-rate optimal within the class $\mathcal{D}_{G}$

$$
\begin{aligned}
\boldsymbol{\mu}_{j}^{T} \boldsymbol{D}^{*} \boldsymbol{\mu}_{j} & \leq d_{j} \quad \forall j \in\{1, \ldots, J\} \\
\frac{1}{2} \log \frac{\left|\boldsymbol{K}_{y}\right|}{\left|\boldsymbol{D}^{*}\right|} & =\inf \left\{\frac{1}{2} \log \frac{\left|\boldsymbol{K}_{y}\right|}{|\boldsymbol{D}|}: \boldsymbol{D} \in \mathcal{D}_{G} \text { and } \boldsymbol{\mu}_{j}^{T} \boldsymbol{D} \boldsymbol{\mu}_{j} \leq d_{j} \forall j \in\{1, \ldots, J\}\right\} .
\end{aligned}
$$

Let $A$ denote the set of constraints that are active at $\boldsymbol{D}^{*}$

$$
A=\left\{j: \boldsymbol{\mu}_{j}^{T} \boldsymbol{D}^{*} \boldsymbol{\mu}_{j}=d_{j}\right\} .
$$

If $|A| \geq 2$, then there exists $i, j$ in $A, i \neq j$, and a nonnegative vector $\boldsymbol{\mu}^{*}$ such that

1. $\boldsymbol{D}^{*}$ is sum-rate optimal for the $\boldsymbol{\mu}^{*}$-sum problem

$$
\frac{1}{2} \log \frac{\left|\boldsymbol{K}_{y}\right|}{\left|\boldsymbol{D}^{*}\right|}=\inf \left\{\frac{1}{2} \log \frac{\left|\boldsymbol{K}_{y}\right|}{|\boldsymbol{D}|}: \boldsymbol{D} \in \mathcal{D}_{G} \text { and } \boldsymbol{\mu}^{* T} \boldsymbol{D} \boldsymbol{\mu}^{*} \leq \boldsymbol{\mu}^{* T} \boldsymbol{D}^{*} \boldsymbol{\mu}^{*}\right\}
$$

2. $\boldsymbol{M}_{2}^{-1} \boldsymbol{\mu}^{*}$ is nonnegative, where

$$
\boldsymbol{M}_{2}=\left[\begin{array}{ll}
\boldsymbol{\mu}_{i} & \boldsymbol{\mu}_{j}
\end{array}\right] .
$$

Proof. Any $\boldsymbol{D} \in \mathcal{D}_{G}$ can be written

$$
\boldsymbol{D}^{-1}=\boldsymbol{K}_{y}^{-1}+\boldsymbol{\Lambda}
$$

for some diagonal and positive semidefinite matrix $\boldsymbol{\Lambda}$. Thus the optimization problem in (52) can be written

$$
\min \quad \frac{1}{2} \log \frac{\left|\boldsymbol{K}_{y}^{-1}+\boldsymbol{\Lambda}\right|}{\left|\boldsymbol{K}_{y}^{-1}\right|}
$$

subject to

$$
\boldsymbol{\mu}_{j}^{T}\left(\boldsymbol{K}_{y}^{-1}+\boldsymbol{\Lambda}\right)^{-1} \boldsymbol{\mu}_{j} \leq d_{j} \quad j \in\{1, \ldots, J\}
$$


where $\boldsymbol{\Lambda}$ ranges over all diagonal and positive semidefinite matrices. By hypothesis, this optimization problem is solved by

$$
\boldsymbol{\Lambda}^{*}=\boldsymbol{D}^{*-1}-\boldsymbol{K}_{y}^{-1} .
$$

Unfortunately, $\boldsymbol{\Lambda}^{*}$ may not be regular [23, p. 309] for this optimization problem. This is an issue because the KKT conditions may not hold at a local minimum that is not regular [23, Example 3.1.1]. We proceed by using a generalization of the KKT conditions called the Fritz John conditions [23, Sec. 3.3.5]. The difference between the Fritz John conditions and the KKT conditions is that the Fritz John conditions include a scalar for the objective in addition to the multipliers for the constraints. The Lagrangian for this optimization problem is

$$
\frac{1}{2} \log \frac{\left|\boldsymbol{K}_{y}^{-1}+\boldsymbol{\Lambda}\right|}{\left|\boldsymbol{K}_{y}^{-1}\right|}-\sum_{j=1}^{J} \nu_{j}\left(d_{j}-\boldsymbol{\mu}_{j}^{T}\left(\boldsymbol{K}_{y}^{-1}+\boldsymbol{\Lambda}\right)^{-1} \boldsymbol{\mu}_{j}\right)-\sum_{j=1}^{2} \nu_{J+j} \mathbf{e}_{j}^{T} \boldsymbol{\Lambda} \mathbf{e}_{j},
$$

where the second summation handles the constraint that $\boldsymbol{\Lambda}$ must be positive semidefinite. Write

$$
\boldsymbol{D}^{*}=\left[\begin{array}{cc}
d_{1}^{*} & \theta^{*} \sqrt{d_{1}^{*} d_{2}^{*}} \\
\theta^{*} \sqrt{d_{1}^{*} d_{2}^{*}} & d_{2}^{*}
\end{array}\right] .
$$

By differentiating the Lagrangian with respect to the two diagonal entries of $\boldsymbol{\Lambda}$ and using the calculations in Appendix H, we can express the Fritz John conditions [23, Prop. 3.3.5] as

$$
\begin{aligned}
& \nu_{0}^{*} d_{1}^{*}=\sum_{j=1}^{J} \nu_{j}^{*}\left(\boldsymbol{\mu}_{j}^{T} \boldsymbol{D}^{*} \mathbf{e}_{1}\right)^{2}+\nu_{J+1}^{*} \\
& \nu_{0}^{*} d_{2}^{*}=\sum_{j=1}^{J} \nu_{j}^{*}\left(\boldsymbol{\mu}_{j}^{T} \boldsymbol{D}^{*} \mathbf{e}_{2}\right)^{2}+\nu_{J+2}^{*}
\end{aligned}
$$

for some $\nu_{0}^{*}, \ldots, \nu_{J+2}^{*}$ that are nonnegative but not all zero. Now since $\boldsymbol{D}^{*} \in \mathcal{D}_{G}^{\circ}$ by hypothesis, the constraint that $\boldsymbol{\Lambda}$ be positive semidefinite is not active at $\Lambda^{*}$. By complimentary slackness, this implies that $\nu_{J+1}^{*}=\nu_{J+2}^{*}=0$. Also by complimentary slackness, $\nu_{j}^{*}=0$ if $j \notin A$, so

$$
\begin{aligned}
& \nu_{0}^{*} d_{1}^{*}=\sum_{j \in A} \nu_{j}^{*}\left(\boldsymbol{\mu}_{j}^{T} \boldsymbol{D}^{*} \mathbf{e}_{1}\right)^{2} \\
& \nu_{0}^{*} d_{2}^{*}=\sum_{j \in A} \nu_{j}^{*}\left(\boldsymbol{\mu}_{j}^{T} \boldsymbol{D}^{*} \mathbf{e}_{2}\right)^{2} .
\end{aligned}
$$

Now if $\nu_{0}^{*}$ were equal to zero, then we would have

$$
\sum_{j \in A} \nu_{j}^{*}\left[\left(\boldsymbol{\mu}_{j}^{T} \boldsymbol{D}^{*} \mathbf{e}_{1}\right)^{2}+\left(\boldsymbol{\mu}_{j}^{T} \boldsymbol{D}^{*} \mathbf{e}_{2}\right)^{2}\right]=0
$$


Since $\boldsymbol{\mu}_{j}$ is unit-norm for all $j$ by assumption, $\left(\boldsymbol{\mu}_{j}^{T} \boldsymbol{D}^{*} \mathbf{e}_{1}\right)^{2}+\left(\boldsymbol{\mu}_{j}^{T} \boldsymbol{D}^{*} \mathbf{e}_{2}\right)^{2}$ must be positive for all $j$. Thus (53) would imply that $\nu_{j}^{*}=0$ for all $j$ in $A$. But this would contradict the condition that at least one of the dual variables $\nu_{0}^{*}, \ldots, \nu_{J+2}^{*}$ is nonzero. It follows that $\nu_{0}^{*}$ is positive and

$$
\begin{aligned}
d_{1}^{*} & =\sum_{j \in A} \frac{\nu_{j}^{*}}{\nu_{0}^{*}}\left(\boldsymbol{\mu}_{j}^{T} \boldsymbol{D}^{*} \mathbf{e}_{1}\right)^{2} \\
d_{2}^{*} & =\sum_{j \in A} \frac{\nu_{j}^{*}}{\nu_{0}^{*}}\left(\boldsymbol{\mu}_{j}^{T} \boldsymbol{D}^{*} \mathbf{e}_{2}\right)^{2} .
\end{aligned}
$$

Thus $\left(d_{1}^{*}, d_{2}^{*}\right)$ lies in the convex cone formed by the points

$$
\left(\left(\boldsymbol{\mu}_{j}^{T} \boldsymbol{D}^{*} \mathbf{e}_{1}\right)^{2},\left(\boldsymbol{\mu}_{j}^{T} \boldsymbol{D}^{*} \mathbf{e}_{2}\right)^{2}\right) \quad j \in A .
$$

By Carathéodory's theorem for convex cones [22, Corollary 17.1.2], there exists $i, j$ in $A, i \neq j$, such that $\left(d_{1}^{*}, d_{2}^{*}\right)$ lies in the convex cone of those two points alone

$$
\begin{aligned}
& d_{1}^{*}=\tilde{\nu}_{i}\left(\boldsymbol{\mu}_{i}^{T} \boldsymbol{D}^{*} \mathbf{e}_{1}\right)^{2}+\tilde{\nu}_{j}\left(\boldsymbol{\mu}_{j}^{T} \boldsymbol{D}^{*} \mathbf{e}_{1}\right)^{2} \\
& d_{2}^{*}=\tilde{\nu}_{i}\left(\boldsymbol{\mu}_{i}^{T} \boldsymbol{D}^{*} \mathbf{e}_{2}\right)^{2}+\tilde{\nu}_{j}\left(\boldsymbol{\mu}_{j}^{T} \boldsymbol{D}^{*} \mathbf{e}_{2}\right)^{2} .
\end{aligned}
$$

Let $\boldsymbol{M}_{2}$ denote the $2 \times 2$ matrix

$$
M_{2}=\left[\begin{array}{ll}
\boldsymbol{\mu}_{i} & \boldsymbol{\mu}_{j}
\end{array}\right] .
$$

By swapping the roles of $\boldsymbol{\mu}_{i}$ and $\boldsymbol{\mu}_{j}$ if necessary, we may assume that $\left|\boldsymbol{M}_{2}\right|>0$.

Let

$$
\boldsymbol{\mu}^{*}=\left[\begin{array}{c}
\sqrt{d_{2}^{*}} \\
\sqrt{d_{1}^{*}}
\end{array}\right] .
$$

By Lemma $4, \boldsymbol{D}^{*}$ is sum-rate optimal for the $\boldsymbol{\mu}^{*}$-sum problem, so it only remains to show that $\boldsymbol{M}_{2}^{-1} \boldsymbol{\mu}^{*}$ is nonnegative.

Consider the matrix

$$
\tilde{\boldsymbol{D}}=\left[\begin{array}{cc}
\left(\boldsymbol{\mu}_{i}^{T} \boldsymbol{D}^{*} \mathbf{e}_{1}\right)^{2} & \left(\boldsymbol{\mu}_{j}^{T} \boldsymbol{D}^{*} \mathbf{e}_{1}\right)^{2} \\
\left(\boldsymbol{\mu}_{i}^{T} \boldsymbol{D}^{*} \mathbf{e}_{2}\right)^{2} & \left(\boldsymbol{\mu}_{j}^{T} \boldsymbol{D}^{*} \mathbf{e}_{2}\right)^{2}
\end{array}\right]
$$

In terms of the components of $\boldsymbol{D}^{*}$ and $\boldsymbol{M}_{2}$, the determinant of $\tilde{\boldsymbol{D}}$ is

$$
\begin{aligned}
|\tilde{\boldsymbol{D}}|=\left(\mu_{i 1} d_{1}^{*}+\mu_{i 2} \theta^{*} \sqrt{d_{1}^{*} d_{2}^{*}}\right)^{2}\left(\mu_{j 1} \theta^{*} \sqrt{d_{1}^{*} d_{2}^{*}}+\mu_{j 2} d_{2}^{*}\right)^{2} & \\
& -\left(\mu_{j 1} d_{1}^{*}+\mu_{j 2} \theta^{*} \sqrt{d_{1}^{*} d_{2}^{*}}\right)^{2}\left(\mu_{i 1} \theta^{*} \sqrt{d_{1}^{*} d_{2}^{*}}+\mu_{i 2} d_{2}^{*}\right)^{2} .
\end{aligned}
$$

By expanding the products and using the fact that $\left|\boldsymbol{M}_{2}\right|>0$, one can show that $|\tilde{\boldsymbol{D}}|>0$. In particular, the conditions in (54) can be written as

$$
\begin{aligned}
{\left[\begin{array}{c}
\tilde{\nu}_{i} \\
\tilde{\nu}_{j}
\end{array}\right] } & =\tilde{\boldsymbol{D}}^{-1}\left[\begin{array}{c}
d_{1}^{*} \\
d_{2}^{*}
\end{array}\right] \\
& =\frac{1}{|\tilde{\boldsymbol{D}}|}\left[\begin{array}{cc}
\left(\boldsymbol{\mu}_{j}^{T} \boldsymbol{D}^{*} \mathbf{e}_{2}\right)^{2} & -\left(\boldsymbol{\mu}_{j}^{T} \boldsymbol{D}^{*} \mathbf{e}_{1}\right)^{2} \\
-\left(\boldsymbol{\mu}_{i}^{T} \boldsymbol{D}^{*} \mathbf{e}_{2}\right)^{2} & \left(\boldsymbol{\mu}_{i}^{T} \boldsymbol{D}^{*} \mathbf{e}_{1}\right)^{2}
\end{array}\right]\left[\begin{array}{c}
d_{1}^{*} \\
d_{2}^{*}
\end{array}\right]
\end{aligned}
$$


Since $\tilde{\nu}_{i}$ and $\tilde{\nu}_{j}$ are nonnegative, this implies that

$$
\begin{aligned}
& d_{1}^{*}\left(\boldsymbol{\mu}_{j}^{T} \boldsymbol{D}^{*} \mathbf{e}_{2}\right)^{2} \geq d_{2}^{*}\left(\boldsymbol{\mu}_{j}^{T} \boldsymbol{D}^{*} \mathbf{e}_{1}\right)^{2} \\
& d_{2}^{*}\left(\boldsymbol{\mu}_{i}^{T} \boldsymbol{D}^{*} \mathbf{e}_{1}\right)^{2} \geq d_{1}^{*}\left(\boldsymbol{\mu}_{i}^{T} \boldsymbol{D}^{*} \mathbf{e}_{2}\right)^{2} .
\end{aligned}
$$

Now (55) can be written

$$
\sqrt{d_{1}^{*}}\left(\mu_{j 1} \theta^{*} \sqrt{d_{1}^{*} d_{2}^{*}}+\mu_{j 2} d_{2}^{*}\right) \geq \sqrt{d_{2}^{*}}\left(\mu_{j 1} d_{1}^{*}+\mu_{j 2} \theta^{*} \sqrt{d_{1}^{*} d_{2}^{*}}\right) .
$$

This can be rearranged to show that

$$
\mu_{j 2} \sqrt{d_{2}^{*}} \geq \mu_{j 1} \sqrt{d_{1}^{*}} .
$$

Likewise, (56) implies

$$
\mu_{i 1} \sqrt{d_{1}^{*}} \geq \mu_{i 2} \sqrt{d_{2}^{*}}
$$

Now

$$
\begin{aligned}
\boldsymbol{M}_{2}^{-1} \boldsymbol{\mu}^{*} & =\frac{1}{\mu_{i 1} \mu_{j 2}-\mu_{i 2} \mu_{j 1}}\left[\begin{array}{cc}
\mu_{j 2} & -\mu_{j 1} \\
-\mu_{i 2} & \mu_{i 1}
\end{array}\right]\left[\begin{array}{c}
\sqrt{d_{2}^{*}} \\
\sqrt{d_{1}^{*}}
\end{array}\right] \\
& =\frac{1}{\mu_{i 1} \mu_{j 2}-\mu_{i 2} \mu_{j 1}}\left[\begin{array}{c}
\mu_{j 2} \sqrt{d_{2}^{*}}-\mu_{j 1} \sqrt{d_{1}^{*}} \\
\mu_{i 1} \sqrt{d_{1}^{*}}-\mu_{i 2} \sqrt{d_{2}^{*}}
\end{array}\right]
\end{aligned}
$$

which is component-wise nonnegative due to (57), (58), and the nonnegativity of $\operatorname{det}\left(\boldsymbol{M}_{2}\right)$.

Lemma 14. Suppose $\left(R_{1}, R_{2}, d_{1}, \ldots, d_{J}\right)$ is strict-sense achievable and there exists a $\boldsymbol{D}^{*}$ in $\mathcal{D}_{G}^{\circ}$ that is sum-rate optimal within the class $\mathcal{D}_{G}$ and meets at least two of the distortion constraints with equality. Then

$$
R_{1}+R_{2} \geq \frac{1}{2} \log \frac{\left|\boldsymbol{K}_{y}\right|}{\left|\boldsymbol{D}^{*}\right|}=R_{\text {sum }}^{\star}\left(d_{1}, \ldots, d_{J}\right) .
$$

Proof. We give an abbreviated proof due to the similarity to the proofs of Lemmas 5 and 6 . From Lemma 13, there exists two constraint vectors $\boldsymbol{\mu}_{i}$ and $\boldsymbol{\mu}_{j}$, $i \neq j$, and a nonnegative vector $\boldsymbol{\mu}^{*}$ such that

(i) $\boldsymbol{\mu}_{i}^{T} \boldsymbol{D}^{*} \boldsymbol{\mu}_{i}=d_{i}$

(ii) $\boldsymbol{\mu}_{j}^{T} \boldsymbol{D}^{*} \boldsymbol{\mu}_{j}=d_{j}$

(iii) $\boldsymbol{D}^{*}$ is sum-rate optimal for the $\boldsymbol{\mu}^{*}$-sum problem, and

(iv) $\boldsymbol{M}_{2}^{-1} \boldsymbol{\mu}^{*}$ is nonnegative, where

$$
\boldsymbol{M}_{2}=\left[\begin{array}{ll}
\boldsymbol{\mu}_{i} & \boldsymbol{\mu}_{j}
\end{array}\right]
$$


For $\theta$ in $(-1,1)$, let

$$
\tilde{\boldsymbol{D}}_{\theta}=\left[\begin{array}{cc}
d_{i} & \theta \sqrt{d_{i} d_{j}} \\
\theta \sqrt{d_{i} d_{j}} & d_{j}
\end{array}\right]
$$

Also define

$$
\tilde{R}_{\text {coop }}(\theta)=\frac{1}{2} \log ^{+} \frac{\left|\boldsymbol{K}_{y}\right|\left|\boldsymbol{M}_{2}\right|^{2}}{\left|\tilde{\boldsymbol{D}}_{\theta}\right|}
$$

and

$$
\begin{aligned}
& \tilde{R}_{\text {sum }}(\theta) \\
& \quad=\inf \left\{\frac{1}{2} \log \frac{\left|\boldsymbol{K}_{y}\right|}{|\boldsymbol{D}|}: \boldsymbol{D} \in \mathcal{D}_{G} \text { and } \boldsymbol{\mu}^{* T} \boldsymbol{D} \boldsymbol{\mu}^{*} \leq \boldsymbol{\mu}^{* T} \boldsymbol{M}_{2}^{-T} \tilde{\boldsymbol{D}}_{\theta} \boldsymbol{M}_{2}^{-1} \boldsymbol{\mu}^{*}\right\} .
\end{aligned}
$$

Now fix some code that achieves $\left(R_{1}, R_{2}, d_{1}, \ldots, d_{J}\right)$. Let $\mathbf{z}^{n}(i)$ denote the decoder's estimate of $\boldsymbol{M}_{2}^{T} \mathbf{y}^{n}(i)$, which we may assume is the conditional expectation of $\boldsymbol{M}_{2}^{T} \mathbf{y}^{n}(i)$ given the received messages. Let

$$
\tilde{\boldsymbol{D}}=\frac{1}{n} \sum_{i=1}^{n} E\left[\left(\boldsymbol{M}_{2}^{T} \mathbf{y}^{n}(i)-\mathbf{z}^{n}(i)\right)\left(\boldsymbol{M}_{2}^{T} \mathbf{y}^{n}(i)-\mathbf{z}^{n}(i)\right)^{T}\right]
$$

denote the average covariance matrix of $\boldsymbol{M}_{2}^{T} \mathbf{y}^{n}-\mathbf{z}^{n}$. Then

$$
\hat{\boldsymbol{D}}=\boldsymbol{M}_{2}^{-T} \tilde{\boldsymbol{D}} \boldsymbol{M}_{2}^{-1}
$$

is the error covariance matrix for the estimate of the source, $\mathbf{y}^{n}$. As in the proof of Lemma $5, \hat{\boldsymbol{D}}$ must be positive definite, and the sum rate of the code must satisfy (c.f. (16))

$$
\begin{aligned}
R_{1}+R_{2} & \geq \frac{1}{2} \log ^{+} \frac{\left|\boldsymbol{K}_{y}\right|}{|\hat{\boldsymbol{D}}|} \\
& =\frac{1}{2} \log ^{+} \frac{\left|\boldsymbol{K}_{y}\right|\left|\boldsymbol{M}_{2}\right|^{2}}{\left|\boldsymbol{M}_{2}^{T} \hat{\boldsymbol{D}} \boldsymbol{M}_{2}\right|} \\
& =\frac{1}{2} \log ^{+} \frac{\left|\boldsymbol{K}_{y}\right|\left|\boldsymbol{M}_{2}\right|^{2}}{|\tilde{\boldsymbol{D}}|} .
\end{aligned}
$$

In particular, $\tilde{\boldsymbol{D}}$ must also be positive definite. Let us write it as

$$
\tilde{\boldsymbol{D}}=\left[\begin{array}{cc}
\tilde{d}_{i} & \tilde{\theta} \sqrt{\tilde{d}_{i} \tilde{d}_{j}} \\
\tilde{\theta} \sqrt{\tilde{d}_{i} \tilde{d}_{j}} & \tilde{d}_{j}
\end{array}\right]
$$

where $\tilde{d}_{i} \leq d_{i}, \tilde{d}_{j} \leq d_{j}$, and $\tilde{\theta} \in(-1,1)$. Now define

$$
\phi=\frac{\tilde{\theta} \sqrt{\tilde{d}_{i} \tilde{d}_{j}}}{\sqrt{d_{i} d_{j}}},
$$


and note that $\phi$ is in $(-1,1)$. Then $\tilde{\boldsymbol{D}} \preceq \tilde{\boldsymbol{D}}_{\phi}$, so it follows that

$$
R_{1}+R_{2} \geq \frac{1}{2} \log ^{+} \frac{\left|\boldsymbol{K}_{y}\right|\left|\boldsymbol{M}_{2}\right|^{2}}{\left|\tilde{\boldsymbol{D}}_{\phi}\right|}=\tilde{R}_{\mathrm{coop}}(\phi) .
$$

On the other hand,

$$
\begin{aligned}
\frac{1}{n} \sum_{i=1}^{n} E\left[\left(\boldsymbol{\mu}^{* T} \mathbf{y}^{n}(i)-\boldsymbol{\mu}^{* T} \boldsymbol{M}_{2}^{-T} \mathbf{z}^{n}(i)\right)^{2}\right] & =\boldsymbol{\mu}^{* T} \boldsymbol{M}_{2}^{-T} \tilde{\boldsymbol{D}} \boldsymbol{M}_{2}^{-1} \boldsymbol{\mu}^{*} \\
& \leq \boldsymbol{\mu}^{* T} \boldsymbol{M}_{2}^{-T} \tilde{\boldsymbol{D}}_{\phi} \boldsymbol{M}_{2}^{-1} \boldsymbol{\mu}^{*}
\end{aligned}
$$

i.e., this code achieves distortion $\boldsymbol{\mu}^{* T} \boldsymbol{M}_{2}^{-T} \tilde{\boldsymbol{D}}_{\phi} \boldsymbol{M}_{2}^{-1} \boldsymbol{\mu}^{*}$ for the $\boldsymbol{\mu}^{*}$-sum problem. Lemma 3 then implies that

$$
R_{1}+R_{2} \geq \tilde{R}_{\mathrm{sum}}(\phi)
$$

It follows that

$$
R_{1}+R_{2} \geq \inf _{\theta \in(-1,1)} \max \left(\tilde{R}_{\text {coop }}(\theta), \tilde{R}_{\text {sum }}(\theta)\right)
$$

Now let

$$
\tilde{\boldsymbol{D}}^{*}=\boldsymbol{M}_{2}^{T} \boldsymbol{D}^{*} \boldsymbol{M}_{2}
$$

which must have diagonal entries $d_{i}$ and $d_{j}$, respectively. Let us write $\tilde{\boldsymbol{D}}^{*}$ as

$$
\tilde{\boldsymbol{D}}^{*}=\left[\begin{array}{cc}
d_{i} & \tilde{\theta}^{*} \sqrt{d_{i} d_{j}} \\
\tilde{\theta}^{*} \sqrt{d_{i} d_{j}} & d_{j}
\end{array}\right] .
$$

Note that we must have $\tilde{\theta}^{*} \in(0,1)$ since $\tilde{\boldsymbol{D}}^{*}$ is positive definite and both $\boldsymbol{M}_{2}$ and $\boldsymbol{D}^{*}$ have positive entries. For $\theta \geq \tilde{\theta}^{*}$, we have

$$
\begin{aligned}
\max \left(\tilde{R}_{\text {coop }}(\theta), \tilde{R}_{\text {sum }}(\theta)\right) \geq \tilde{R}_{\text {coop }}(\theta) \geq \tilde{R}_{\text {coop }}\left(\tilde{\theta}^{*}\right) & =\frac{1}{2} \log ^{+} \frac{\left|\boldsymbol{K}_{y}\right|\left|\boldsymbol{M}_{2}\right|^{2}}{\left|\tilde{\boldsymbol{D}}^{*}\right|} \\
& =\frac{1}{2} \log ^{+} \frac{\left|\boldsymbol{K}_{y}\right|}{\left|\boldsymbol{D}^{*}\right|} .
\end{aligned}
$$

Since both components of $\boldsymbol{M}_{2}^{-1} \boldsymbol{\mu}^{*}$ are nonnegative, it follows that

$$
\boldsymbol{\mu}^{* T} \boldsymbol{M}_{2}^{-T} \tilde{\boldsymbol{D}}_{\theta} \boldsymbol{M}_{2}^{-1} \boldsymbol{\mu}^{*}
$$

is nondecreasing in $\theta$, which implies that $\tilde{R}_{\text {sum }}(\cdot)$ is nonincreasing. Thus if $\theta \leq \tilde{\theta}^{*}$

$$
\max \left(\tilde{R}_{\text {coop }}(\theta), \tilde{R}_{\text {sum }}(\theta)\right) \geq \tilde{R}_{\text {sum }}(\theta) \geq \tilde{R}_{\text {sum }}\left(\tilde{\theta}^{*}\right) .
$$

But

$$
\tilde{R}_{\text {sum }}\left(\tilde{\theta}^{*}\right)=\inf \left\{\frac{1}{2} \log \frac{\left|\boldsymbol{K}_{y}\right|}{|\boldsymbol{D}|}: \boldsymbol{D} \in \mathcal{D}_{G} \text { and } \boldsymbol{\mu}^{* T} \boldsymbol{D} \boldsymbol{\mu}^{*} \leq \boldsymbol{\mu}^{* T} \boldsymbol{M}_{2}^{-1} \tilde{\boldsymbol{D}}^{*} \boldsymbol{M}_{2}^{-1} \boldsymbol{\mu}^{*}\right\}
$$


and

$$
\boldsymbol{M}_{2}^{-T} \tilde{\boldsymbol{D}}^{*} \boldsymbol{M}_{2}^{-1}=\boldsymbol{D}^{*} .
$$

Since $\boldsymbol{D}^{*}$ solves the $\boldsymbol{\mu}^{*}$-sum problem, this implies

$$
\tilde{R}_{\text {sum }}\left(\tilde{\theta}^{*}\right)=\frac{1}{2} \log \frac{\left|\boldsymbol{K}_{y}\right|}{\left|\boldsymbol{D}^{*}\right|} .
$$

It follows that

$$
\inf _{\theta \in(-1,1)} \max \left(\tilde{R}_{\text {coop }}(\theta), \tilde{R}_{\text {sum }}(\theta)\right)=\frac{1}{2} \log \frac{\left|\boldsymbol{K}_{y}\right|}{\left|\boldsymbol{D}^{*}\right|} .
$$

Combining this with (59) yields the desired conclusion.

We are now in a position to complete the proof of Theorem 2.

Proof of Theorem 2. We only need to show that the rate region is contained in $\mathcal{R}_{\text {sum }}^{\star}\left(d_{1}, \ldots, d_{J}\right)$. Lemmas 11,12 , and 14 together imply that if

$$
\left(R_{1}, R_{2}, d_{1}, \ldots, d_{J}\right)
$$

is strict-sense achievable, then

$$
R_{1}+R_{2} \geq \inf \left\{\frac{1}{2} \log \frac{\left|\boldsymbol{K}_{y}\right|}{|\boldsymbol{D}|}: \boldsymbol{D} \in \mathcal{D}_{G} \text { and } \boldsymbol{\mu}_{i}^{T} \boldsymbol{D} \boldsymbol{\mu}_{i} \leq d_{j} \forall j \in\{1, \ldots, J\}\right\} .
$$

It is readily verified that the right-hand side is lower-semicontinuous in $d_{1}, \ldots, d_{J}$. It follows that (60) also holds if $\left(R_{1}, R_{2}, d_{1}, \ldots, d_{J}\right)$ are achievable. This implies the desired conclusion.

\section{G Converse for Many Sources}

In this appendix we prove Theorem 3 . Recall we are assuming that the covariance matrix $\boldsymbol{K}_{y}$ has the form

$$
\boldsymbol{K}_{y}=\left[\begin{array}{cccc}
1 & \rho & \cdots & \rho \\
\rho & 1 & \cdots & \rho \\
\vdots & \vdots & \ddots & \vdots \\
\rho & \rho & \cdots & 1
\end{array}\right] .
$$

for some $0<\rho<1$ and all of the distortion constraints are equal to $d$. Our goal is to show that the minimum sum rate, $R_{\mathrm{sum}}^{\star}(d)$, equals

$$
\inf \left\{\frac{1}{2} \log \frac{\left|\boldsymbol{K}_{y}\right|}{|\boldsymbol{D}|}: \boldsymbol{D} \in \mathcal{D}_{G} \text { and } \mathbf{e}_{\ell}^{T} \boldsymbol{D} \mathbf{e}_{\ell} \leq d \forall \ell \in\{1, \ldots, L\}\right\},
$$

and that this infimum is achieved by a $\boldsymbol{D} \in \mathcal{D}_{G}$ of the form

$$
\boldsymbol{D}^{-1}=\boldsymbol{K}_{y}^{-1}+\lambda \boldsymbol{I}
$$


for some $\lambda \geq 0$. The conclusion is obvious if $d \geq 1$, so we will assume that $0<d<1$. Using the matrix inversion lemma [24, p. 50], the matrix inversions in (61) can be computed explicitly

$$
\boldsymbol{D}=\frac{1-\rho}{1+\lambda(1-\rho)}\left[\boldsymbol{I}+\frac{\rho \mathbf{1 1}^{T}}{1-\rho+\lambda(1-\rho)(1-\rho+\rho L)}\right] .
$$

It follows that there is a unique $\boldsymbol{D} \in \mathcal{D}_{G}$ of the form in (61) with all of the diagonal entries equal to $d$. Let us call this matrix $\boldsymbol{D}^{*}$. Note that $\boldsymbol{D}^{*}$ must be of the form

$$
\boldsymbol{D}^{*}=d\left[\begin{array}{cccc}
1 & \theta^{*} & \ldots & \theta^{*} \\
\theta^{*} & 1 & \ldots & \theta^{*} \\
\vdots & \vdots & \ddots & \vdots \\
\theta^{*} & \theta^{*} & \ldots & 1
\end{array}\right]
$$

for some $\theta^{*}>0$. Since $\boldsymbol{D}^{*}$ must be positive definite, it follows that $\theta^{*}<1$. Now the inequalities

$$
\begin{aligned}
R_{\text {sum }}^{\star}(d) & \leq \inf \left\{\frac{1}{2} \log \frac{\left|\boldsymbol{K}_{y}\right|}{|\boldsymbol{D}|}: \boldsymbol{D} \in \mathcal{D}_{G} \text { and } \mathbf{e}_{\ell}^{T} \boldsymbol{D} \mathbf{e}_{\ell} \leq d \forall \ell \in\{1, \ldots, L\}\right\} \\
& \leq \frac{1}{2} \log \frac{\left|\boldsymbol{K}_{y}\right|}{\left|\boldsymbol{D}^{*}\right|}
\end{aligned}
$$

are clear, so it suffices to show that

$$
\frac{1}{2} \log \frac{\left|\boldsymbol{K}_{y}\right|}{\left|\boldsymbol{D}^{*}\right|} \leq R_{\mathrm{sum}}^{\star}(d)
$$

For $\theta$ in $(-1 /(L-1), 1)$ let

$$
\boldsymbol{D}_{\theta}=d\left[\begin{array}{cccc}
1 & \theta & \ldots & \theta \\
\theta & 1 & \ldots & \theta \\
\vdots & \vdots & \ddots & \vdots \\
\theta & \theta & \ldots & 1
\end{array}\right]
$$

and note that $\boldsymbol{D}_{\theta}$ is positive definite for each $\theta$. Then define

$$
R_{\text {coop }}(\theta)=\frac{1}{2} \log ^{+} \frac{\left|\boldsymbol{K}_{y}\right|}{\left|\boldsymbol{D}_{\theta}\right|}=\frac{1}{2} \log ^{+} \frac{\left|\boldsymbol{K}_{y}\right|}{d^{L}(1-\theta)^{L-1}(1-\theta+L \theta)} .
$$

Next consider the problem of reproducing the sum of the sources, $\mathbf{1}^{T} \mathbf{y}$, at the decoder. By following the proof of Lemma 8, one can show that this problem is equivalent to the $\mathrm{CEO}$ problem

$$
y_{\ell}=x+n_{\ell} \quad \ell \in\{1, \ldots, L\}
$$

where $x, n_{1}, \ldots, n_{L}$ are zero-mean, Gaussian, and independent, and $x$ has variance $\rho$. It follows from existing results that the separation-based scheme achieves 
the entire rate region, and in particular it is sum-rate optimal $[8,10]$. Thus the sum rate for the $\mathbf{1}$-sum problem with distortion constraint $\mathbf{1}^{T} \boldsymbol{D}_{\theta} \mathbf{1}$ is given by

$$
R_{\text {sum }}(\theta):=\inf \left\{\frac{1}{2} \log \frac{\left|\boldsymbol{K}_{y}\right|}{|\boldsymbol{D}|}: \boldsymbol{D} \in \mathcal{D}_{G} \text { and } \mathbf{1}^{T} \boldsymbol{D} \mathbf{1} \leq \mathbf{1}^{T} \boldsymbol{D}_{\theta} \mathbf{1}\right\} .
$$

In fact, the CEO results imply that the infimum in (64) will be achieved by a $\boldsymbol{D} \in \mathcal{D}_{G}$ of the form in (61). In particular, we have

$$
R_{\text {sum }}\left(\theta^{*}\right)=\frac{1}{2} \log \frac{\left|\boldsymbol{K}_{y}\right|}{\left|\boldsymbol{D}^{*}\right|} .
$$

Lemma 15. If $\left(R_{1}, \ldots, R_{L}, d, \ldots, d\right)$ is strict-sense achievable, then

$$
\sum_{\ell=1}^{L} R_{\ell} \geq \inf _{\theta \in(-1 /(L-1), 1)} \max \left(R_{\text {coop }}(\theta), R_{\text {sum }}(\theta)\right) .
$$

Proof. By hypothesis, there exists a code $\left(f_{1}^{(n)}, \ldots, f_{L}^{(n)}, \varphi_{1}^{(n)}, \ldots, \varphi_{L}^{(n)}\right)$ such that

$$
\begin{aligned}
R_{\ell} & \geq \frac{1}{n} \log M_{\ell}^{(n)} \quad \text { for all } \ell \text { in }\{1, \ldots, L\} \\
d & \geq \frac{1}{n} \sum_{i=1}^{n} E\left[\left(y_{\ell}^{n}(i)-\hat{y}_{\ell}^{n}(i)\right)^{2}\right] \quad \text { for all } \ell \text { in }\{1, \ldots, L\} .
\end{aligned}
$$

From this code, we construct a new code with block length $N:=L ! \cdot n$ by timesharing among all $L$ ! permutations of the sources. For this new code, the rates are symmetric

$$
\frac{1}{L} \sum_{\ell=1}^{L} R_{\ell} \geq \frac{1}{N} \log M_{\ell}^{(N)} \quad \text { for all } \ell \text { in }\{1, \ldots, L\}
$$

and

$$
d \geq \frac{1}{N} \sum_{i=1}^{N} E\left[\left(y_{\ell}^{N}(i)-\hat{y}_{\ell}^{N}(i)\right)^{2}\right] \text { for all } \ell \text { in }\{1, \ldots, L\}
$$

Let

$$
\hat{\boldsymbol{D}}=\frac{1}{N} \sum_{i=1}^{N} E\left[\left(\mathbf{y}^{N}(i)-\hat{\mathbf{y}}^{N}(i)\right)\left(\mathbf{y}^{N}(i)-\hat{\mathbf{y}}^{N}(i)\right)^{T}\right]
$$

denote the error covariance matrix of the code. By the symmetry of the time sharing, $\hat{\boldsymbol{D}}$ must have the form

$$
\hat{\boldsymbol{D}}=\hat{d}\left[\begin{array}{cccc}
1 & \phi & \cdots & \phi \\
\phi & 1 & \cdots & \phi \\
\vdots & \vdots & \ddots & \vdots \\
\phi & \phi & \cdots & 1
\end{array}\right]
$$


for some $\hat{d} \leq d$. Following the calculation at the beginning of the proof of Lemma 5 , one can show that $\hat{\boldsymbol{D}}$ is positive definite, which implies that $-1 /(L-$ 1) $<\phi<1$, and

$$
\sum_{\ell=1}^{L} R_{\ell} \geq \frac{1}{2} \log ^{+} \frac{\left|\boldsymbol{K}_{y}\right|}{|\hat{\boldsymbol{D}}|} .
$$

But $\hat{\boldsymbol{D}} \preceq \boldsymbol{D}_{\phi}$, so this implies

$$
\sum_{\ell=1}^{L} R_{\ell} \geq \frac{1}{2} \log \frac{\left|\boldsymbol{K}_{y}\right|}{\left|\boldsymbol{D}_{\phi}\right|}=R_{\text {coop }}(\phi) .
$$

Since the code has error covariance matrix $\hat{\boldsymbol{D}}$, the distortion it achieves for the $\mathbf{1}$-sum problem is at most $\mathbf{1}^{T} \hat{\boldsymbol{D}} \mathbf{1} \leq \mathbf{1}^{T} \boldsymbol{D}_{\phi} \mathbf{1}$. It follows that

$$
\sum_{\ell=1}^{L} R_{\ell} \geq R_{\mathrm{sum}}(\phi)
$$

Combining this with (67) gives

$$
\sum_{\ell=1}^{L} R_{\ell} \geq \max \left(R_{\text {sum }}(\phi), R_{\text {coop }}(\phi)\right) .
$$

The conclusion follows by taking the infimum over $\phi$ in $(-1 /(L-1), 1)$

Next we evaluate the infimum in (66). Recall that $\theta^{*}$ is defined by (63).

\section{Lemma 16.}

$$
\inf _{\theta \in(-1 /(L-1), 1)} \max \left(R_{\text {coop }}(\theta), R_{\text {sum }}(\theta)\right)=\frac{1}{2} \log \frac{\left|\boldsymbol{K}_{y}\right|}{\left|\boldsymbol{D}^{*}\right|} .
$$

Proof. By differentiating, one can verify that $R_{\text {coop }}(\cdot)$ is nondecreasing on $(0,1)$. Then if $\theta \geq \theta^{*}$, we have

$$
\max \left(R_{\text {coop }}(\theta), R_{\text {sum }}(\theta)\right) \geq R_{\text {coop }}(\theta) \geq R_{\text {coop }}\left(\theta^{*}\right)=\frac{1}{2} \log \frac{\left|\boldsymbol{K}_{y}\right|}{\left|\boldsymbol{D}^{*}\right|} .
$$

On the other hand, if $\theta \leq \theta^{*}$, then since $R_{\text {sum }}(\cdot)$ is nonincreasing

$$
\max \left(R_{\text {coop }}(\theta), R_{\text {sum }}(\theta)\right) \geq R_{\text {sum }}(\theta) \geq R_{\text {sum }}\left(\theta^{*}\right)=\frac{1}{2} \log \frac{\left|\boldsymbol{K}_{y}\right|}{\left|\boldsymbol{D}^{*}\right|},
$$

where we have used (65).

Theorem 3 now follows from a continuity argument similar to the one used in the proof of Theorem 1 . 


\section{H Derivatives}

Let $\boldsymbol{D}\left(\lambda_{1}, \lambda_{2}\right)$ denote the matrix in $\mathcal{D}_{G}$ defined by

$$
\boldsymbol{D}\left(\lambda_{1}, \lambda_{2}\right)=\left(\boldsymbol{K}_{y}^{-1}+\left[\begin{array}{cc}
\lambda_{1} & 0 \\
0 & \lambda_{2}
\end{array}\right]\right)^{-1} .
$$

In this appendix we compute the derivatives of

$$
\log \left|\boldsymbol{D}\left(\lambda_{1}, \lambda_{2}\right)\right|
$$

and

$$
\boldsymbol{\mu}^{T} \boldsymbol{D}\left(\lambda_{1}, \lambda_{2}\right) \boldsymbol{\mu}
$$

with respect to $\lambda_{1}$ and $\lambda_{2}$. Write

$$
\boldsymbol{\Lambda}=\left[\begin{array}{cc}
\lambda_{1} & 0 \\
0 & \lambda_{2}
\end{array}\right]
$$

Then

$$
\begin{aligned}
\frac{\partial \log \left|\boldsymbol{D}\left(\lambda_{1}, \lambda_{2}\right)\right|}{\partial \lambda_{1}} & =-\lim _{\delta \rightarrow 0} \frac{\log \left|\boldsymbol{K}_{y}^{-1}+\boldsymbol{\Lambda}+\delta \mathbf{e}_{1} \mathbf{e}_{1}^{T}\right|-\log \left|\boldsymbol{K}_{y}^{-1}+\boldsymbol{\Lambda}\right|}{\delta} \\
& =-\lim _{\delta \rightarrow 0} \frac{\log \left|\boldsymbol{I}+\delta \mathbf{e}_{1} \mathbf{e}_{1}^{T} \boldsymbol{D}\left(\lambda_{1}, \lambda_{2}\right)\right|}{\delta} \\
& =-\lim _{\delta \rightarrow 0} \frac{\log \left(1+\delta \mathbf{e}_{1}^{T} \boldsymbol{D}\left(\lambda_{1}, \lambda_{2}\right) \mathbf{e}_{1}\right)}{\delta} \\
& =-\log (e) \mathbf{e}_{1}^{T} \boldsymbol{D}\left(\lambda_{1}, \lambda_{2}\right) \mathbf{e}_{1} .
\end{aligned}
$$

Similarly,

$$
\frac{\partial \log \left|\boldsymbol{D}\left(\lambda_{1}, \lambda_{2}\right)\right|}{\partial \lambda_{2}}=-\log (e) \mathbf{e}_{2}^{T} \boldsymbol{D}\left(\lambda_{1}, \lambda_{2}\right) \mathbf{e}_{2} .
$$

Also

$$
\begin{aligned}
& \frac{\partial \boldsymbol{\mu}^{T} \boldsymbol{D}\left(\lambda_{1}, \lambda_{2}\right) \boldsymbol{\mu}}{\partial \lambda_{1}} \\
& =\lim _{\delta \rightarrow 0} \frac{\boldsymbol{\mu}^{T}\left(\left(\boldsymbol{K}_{y}^{-1}+\boldsymbol{\Lambda}+\delta \mathbf{e}_{1} \mathbf{e}_{1}^{T}\right)^{-1}-\left(\boldsymbol{K}_{y}^{-1}+\boldsymbol{\Lambda}\right)^{-1}\right) \boldsymbol{\mu}}{\delta} \\
& =-\lim _{\delta \rightarrow 0} \boldsymbol{\mu}^{T}\left(\boldsymbol{D}\left(\lambda_{1}, \lambda_{2}\right) \mathbf{e}_{1}\left(1+\delta \mathbf{e}_{1}^{T} \boldsymbol{D}\left(\lambda_{1}, \lambda_{2}\right) \mathbf{e}_{1}\right)^{-1} \mathbf{e}_{1}^{T} \boldsymbol{D}\left(\lambda_{1}, \lambda_{2}\right)\right) \boldsymbol{\mu} \\
& =-\left(\boldsymbol{\mu}^{T} \boldsymbol{D}\left(\lambda_{1}, \lambda_{2}\right) \mathbf{e}_{1}\right)^{2}
\end{aligned}
$$

where to obtain the second equation we have used the matrix inversion lemma. Similarly,

$$
\frac{\partial \boldsymbol{\mu}^{T} \boldsymbol{D}\left(\lambda_{1}, \lambda_{2}\right) \boldsymbol{\mu}}{\partial \lambda_{2}}=-\left(\boldsymbol{\mu}^{T} \boldsymbol{D}\left(\lambda_{1}, \lambda_{2}\right) \mathbf{e}_{2}\right)^{2} .
$$




\section{References}

[1] D. Slepian and J. K. Wolf, "Noiseless coding of correlated information sources," IEEE Trans. Inf. Theory, vol. 19, no. 4, pp. 471-480, July 1973.

[2] T. Berger, "Multiterminal source coding," in The Information Theory Approach to Communications, ser. CISM Courses and Lectures, G. Longo, Ed. Springer-Verlag, 1978, vol. 229, pp. 171-231.

[3] S.-Y. Tung, "Multiterminal source coding," Ph.D. dissertation, School of Electrical Engineering, Cornell University, Ithaca, NY, May 1978.

[4] R. Zamir and T. Berger, "Multiterminal source coding with high resolution," IEEE Trans. Inf. Theory, vol. 45, no. 1, pp. 106-117, Jan. 1999.

[5] Y. Oohama, "Gaussian multiterminal source coding," IEEE Trans. Inf. Theory, vol. 43, no. 6, pp. 1912-1923, Nov. 1997.

[6] T. Berger, Z. Zhang, and H. Viswanathan, "The CEO problem," IEEE Trans. Inf. Theory, vol. 42, no. 3, pp. 887-902, May 1996.

[7] M. Gastpar, "The Wyner-Ziv problem with multiple sources," IEEE Trans. Inf. Theory, vol. 50, no. 11, pp. 2762-2768, Nov. 2004.

[8] Y. Oohama, "Rate-distortion theory for Gaussian multiterminal source coding systems with several side informations at the decoder," IEEE Trans. Inf. Theory, vol. 51, no. 7, pp. 2577-2593, July 2005.

[9] H. Viswanathan and T. Berger, "The quadratic Gaussian CEO problem," IEEE Trans. Inf. Theory, vol. 43, no. 5, pp. 1549-1559, Sept. 1997.

[10] V. Prabhakaran, D. Tse, and K. Ramchandran, "Rate region of the quadratic Gaussian CEO problem," in IEEE Int. Symp. Inf. Theor. Proc., 2004 , p. 117.

[11] A. B. Wagner and V. Anantharam, "An improved outer bound for the multiterminal source-coding problem," in IEEE Int. Symp. Inf. Theor. Proc., 2005, pp. 1406-1410.

[12] _ , "An infeasibility result for the multiterminal source-coding problem," arXiv:cs.IT/0511103.

[13] R. G. Gallager, Information Theory and Reliable Communication. New York: John Wiley \& Sons, 1968.

[14] A. Lapidoth, "On the role of mismatch in rate distortion theory," IEEE Trans. Inf. Theory, vol. 43, no. 1, pp. 38-47, Jan. 1997.

[15] T. M. Cover and J. A. Thomas, Elements of Information Theory. New York: John Wiley \& Sons, 1991. 
[16] R. A. Horn and C. R. Johnson, Matrix Analysis. Cambridge University Press, 1985.

[17] Y. Oohama, "Rate distortion region for separate coding of correlated Gaussian remote observations," in Proc. 43rd Annual Allerton Conference, 2005, pp. 2237-2246.

[18] L. Ozarow, "On a source-coding problem with two channels and three receivers," Bell Syst. Tech. J., vol. 59, no. 10, pp. 1909-1921, Dec. 1980.

[19] H. Wang and P. Viswanath, "Vector Gaussian multiple description with individual and central receivers," IEEE Trans. Inf. Theory, vol. 53, no. 6, pp. 2133-2153, June 2007.

[20] S. Tavildar, P. Viswanath, and A. B. Wagner, "The Gaussian many-helpone distributed source coding problem," in Proc. IEEE Inf. Theory Workshop, 2006, pp. 596-600.

[21] S. Boyd and L. Vandenberghe, Convex Optimization. Cambridge University Press, 2004.

[22] R. T. Rockafellar, Convex Analysis. Princeton University Press, 1970.

[23] D. P. Bertsekas, Nonlinear Programming, 2nd ed. Belmont, Mass.: Athena Scientific, 1999.

[24] G. H. Golub and C. F. V. Loan, Matrix Computations, 3rd ed. Baltimore: The Johns Hopkins University Press, 1996. 


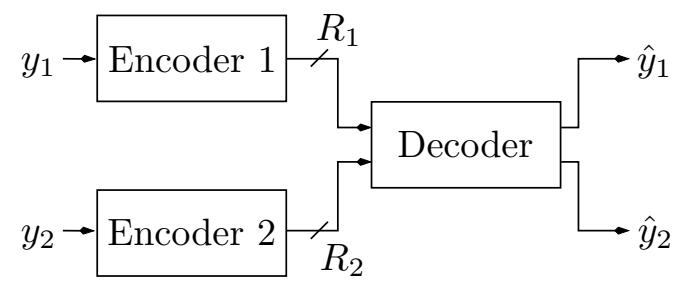

Figure 1: The two-encoder source-coding problem.

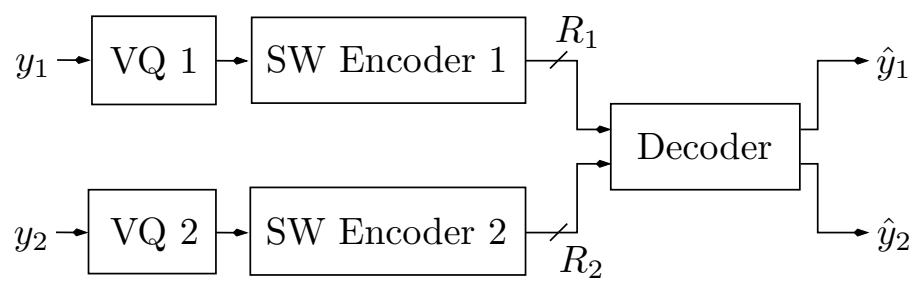

Figure 2: A natural architecture that separates the analog and digital aspects of the compression.

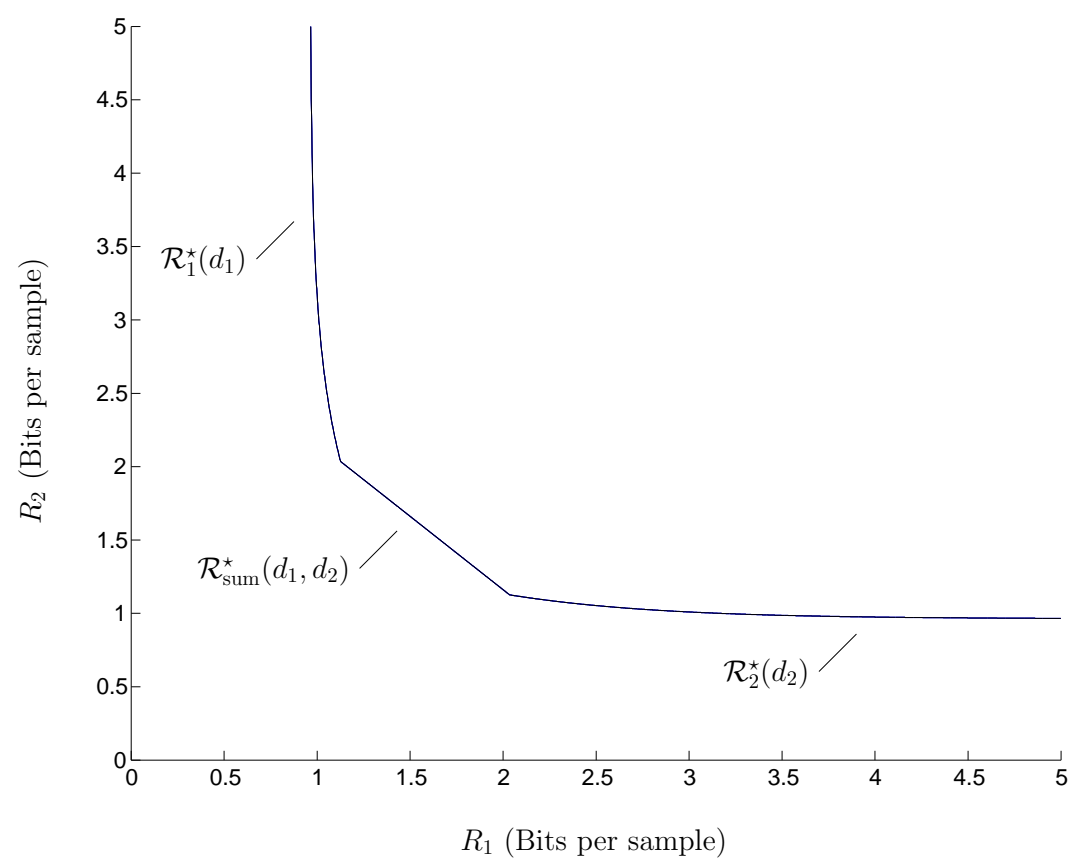

Figure 3: The rate region for $\rho=0.9$ and $d_{1}=d_{2}=0.05$. 


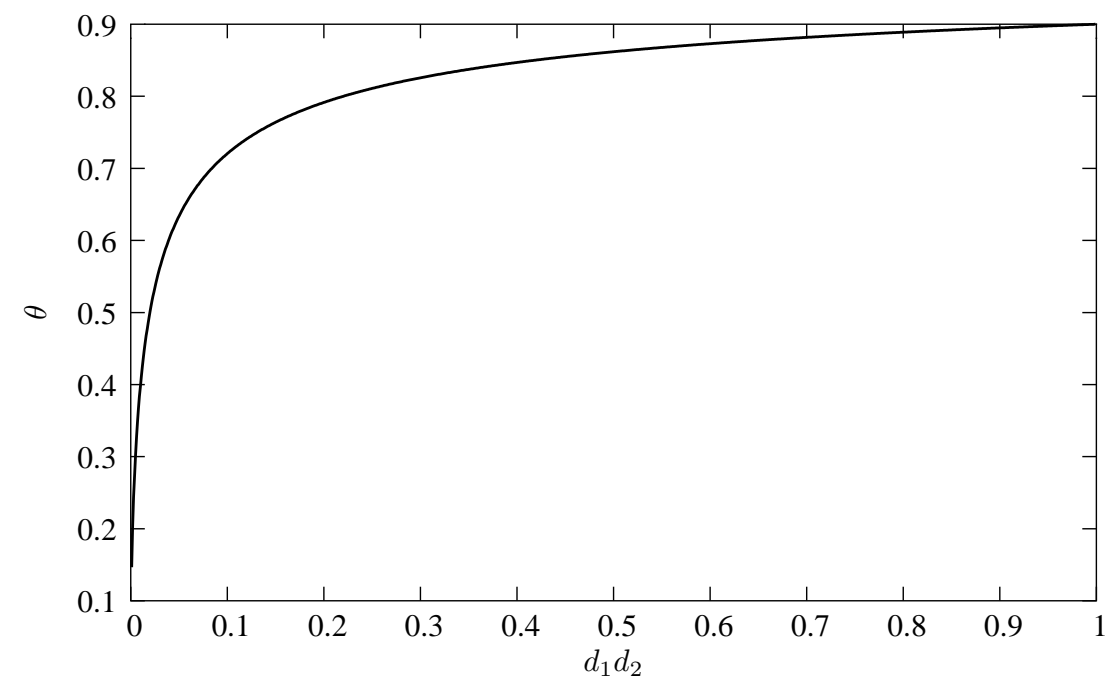

Figure 4: Dependence of the product $d_{1} d_{2}$ on the error correlation coefficient $\theta$ for $\rho=0.9$. 


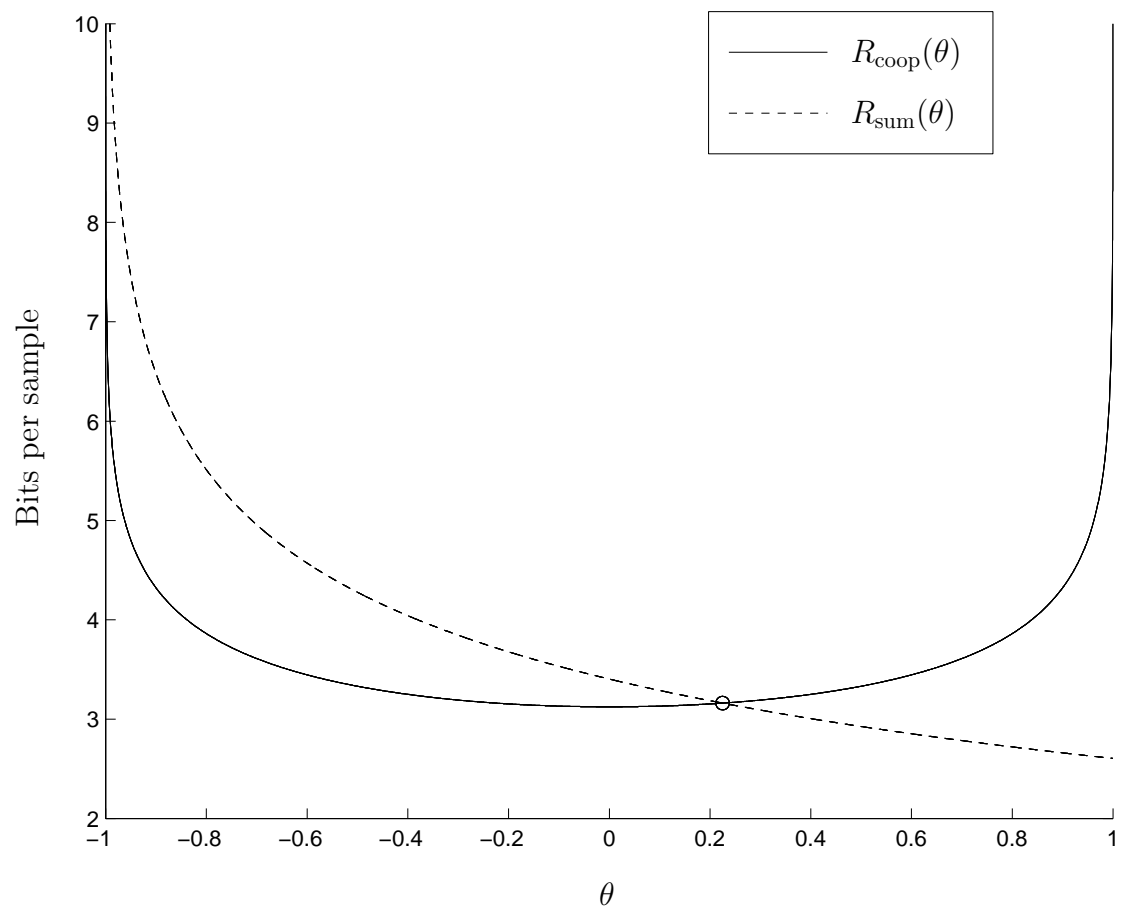

Figure 5: $R_{\text {coop }}(\cdot)$ and $R_{\text {sum }}(\cdot)$ for the case $\rho=0.9$ and $d_{1}=d_{2}=0.05$. The plot for $R_{\text {sum }}(\cdot)$ was generated using the convex optimization formulation of the sum rate for the $\boldsymbol{\mu}$-sum problem given in Appendix C. The circled point at which the two functions intersect is the min-max and equals the sum rate. 


\section{original problem}

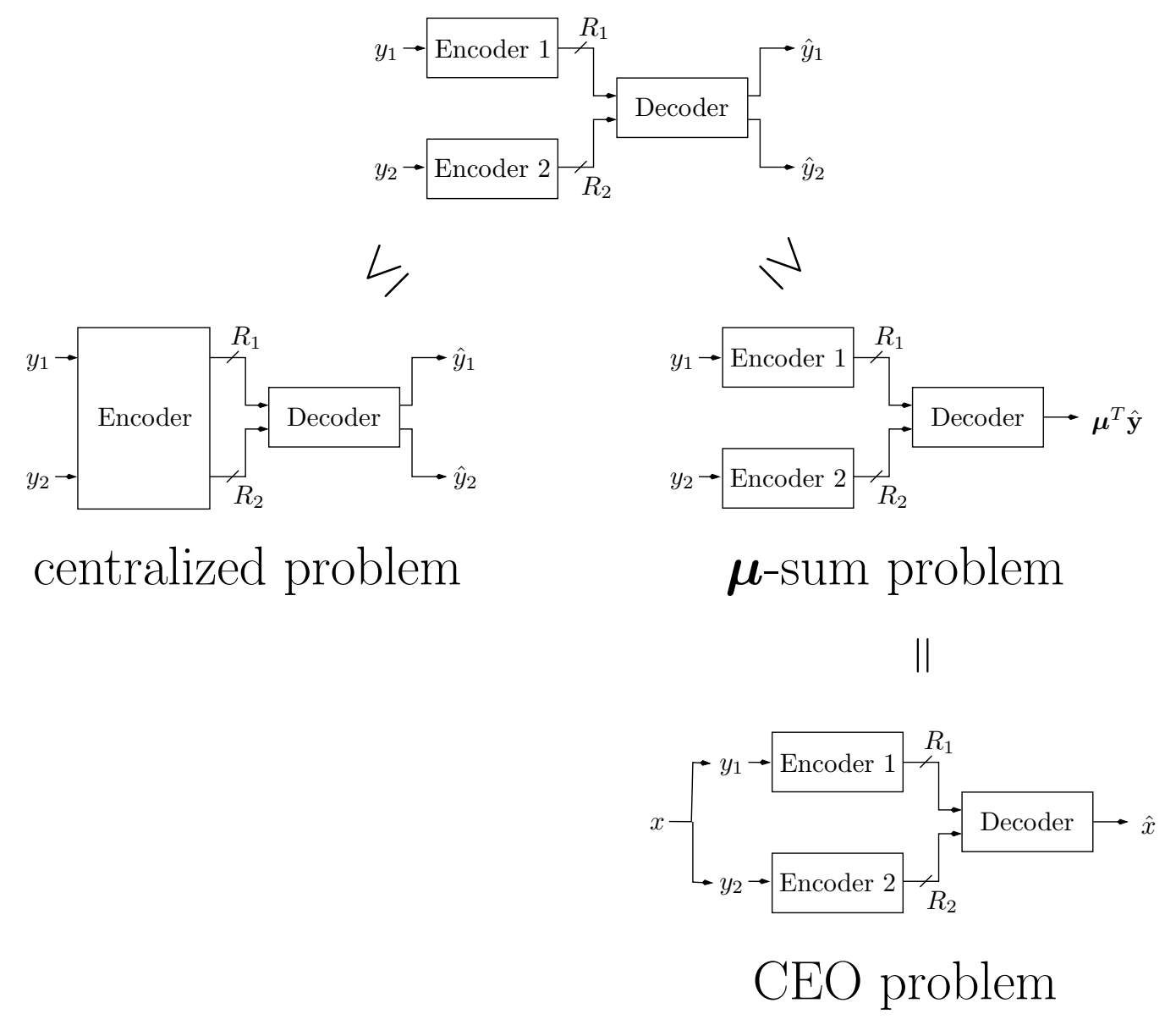

Figure 6: Graphical depiction of the lower bound argument. 


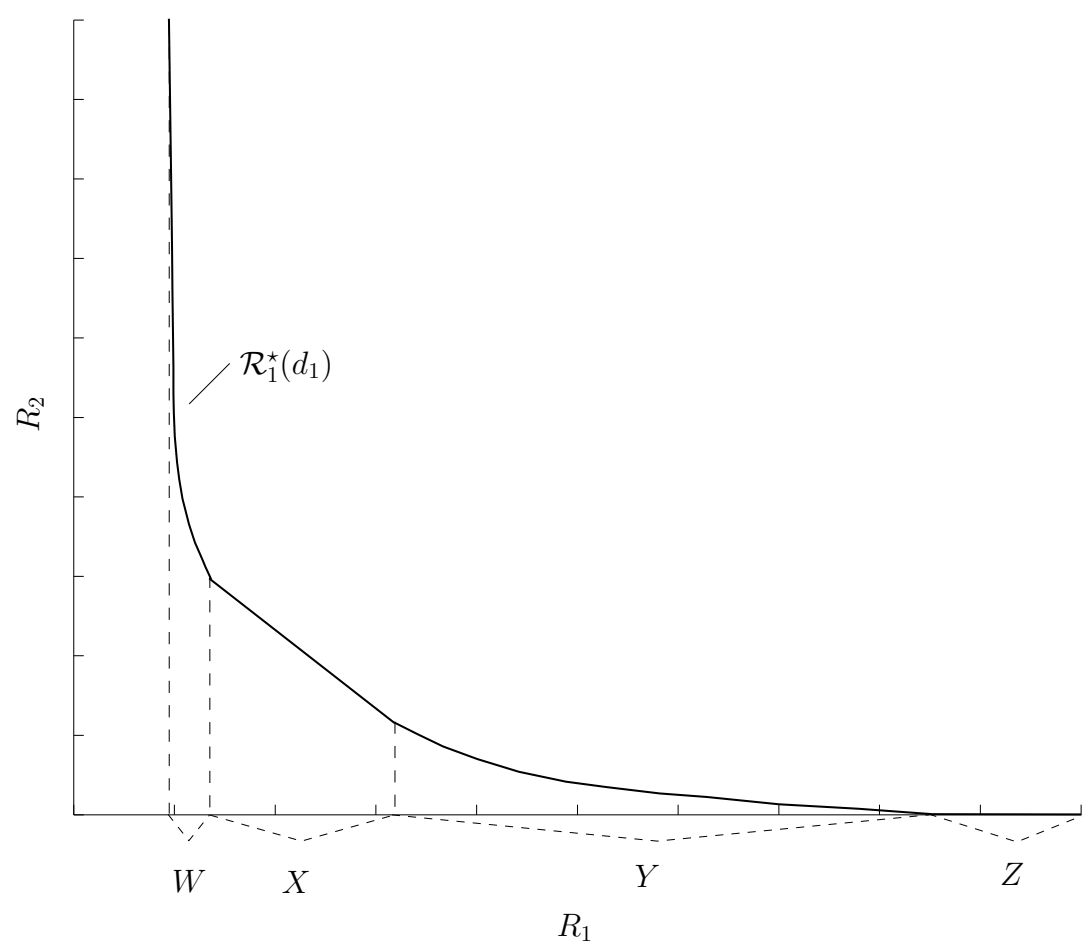

Figure 7: An example illustrating the definitions of $W, X, Y$, and $Z$ in Appendix E. 


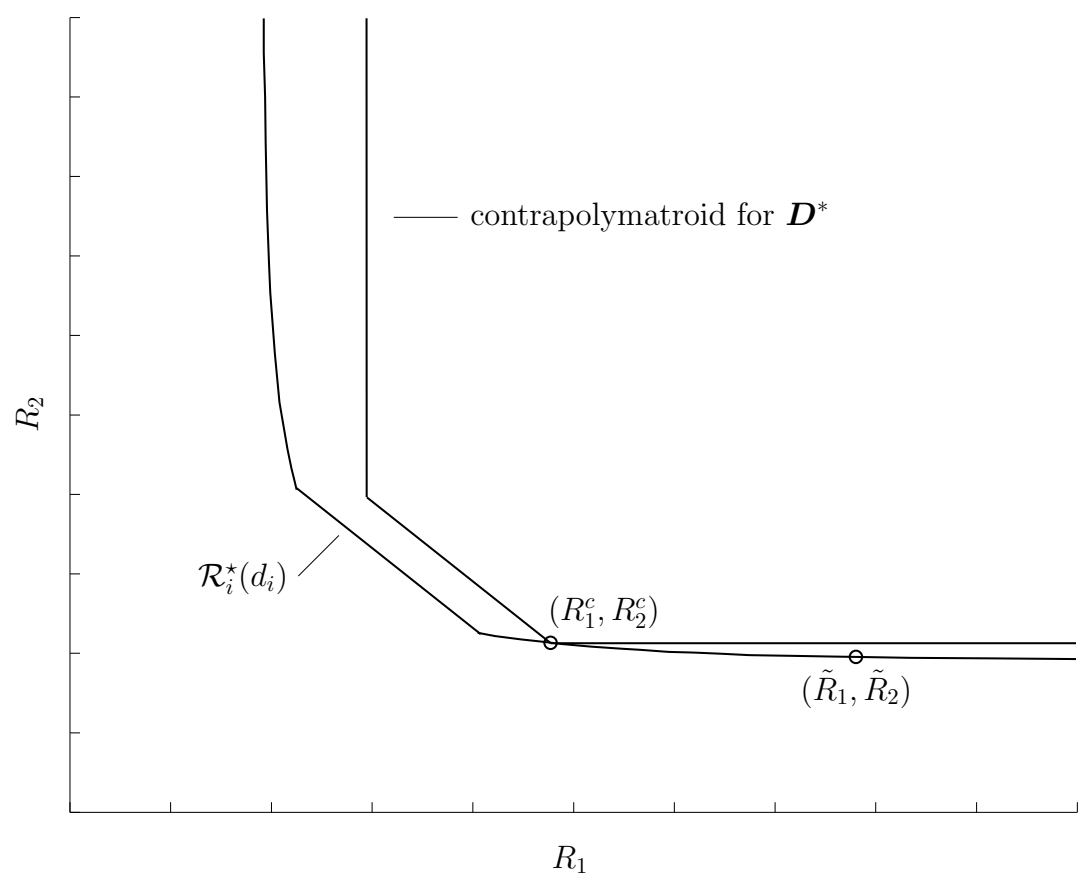

Figure 8: Illustration of $\boldsymbol{M}$-sums achievability argument.

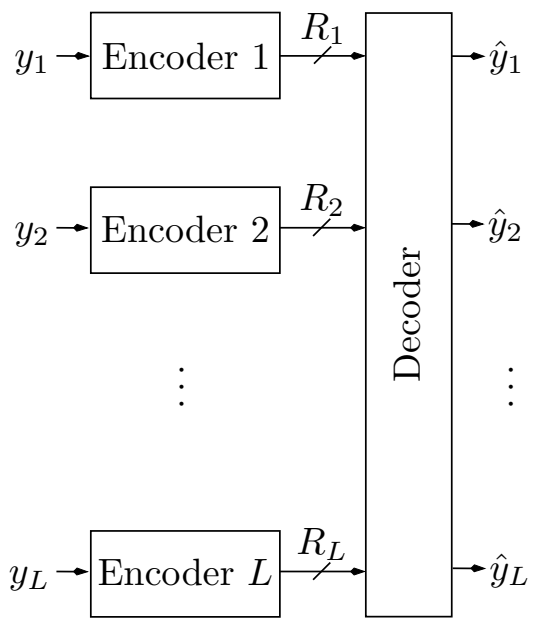

Figure 9: Setup for the many sources problem. 